\title{
An Evertse-Ferretti Nevanlinna constant and its consequences
}

\author{
Min $\mathrm{Ru}^{1}$ (D) Paul Vojta ${ }^{2}$
}

Received: 24 April 2020 / Accepted: 28 June 2021 / Published online: 2 August 2021

(C) The Author(s) 2021

\begin{abstract}
In this paper, we introduce the notion of an Evertse-Ferretti Nevanlinna constant and compare it with the birational Nevanlinna constant introduced by the authors in a recent joint paper. We then use it to recover several previously known results. This includes a 1999 example of Faltings from his Baker's Garden article. We also extend the theory of these Nevanlinna constants to what we call "multidivisor Nevanlinna constants," which allow the proximity function to involve the maximum of Weil functions for finitely many divisors.
\end{abstract}

Keywords Nevanlinna constant - Schmidt's Subspace Theorem · Integral points · Diophantine approximation $\cdot$ Nevanlinna theory $\cdot$ Filtration method

Mathematics Subject Classification 32H30 - 11J97

Communicated by Adrian Constantin.

The first author is supported in part by the Simons Foundation grant award \#531604. Parts of this work were performed while the second author enjoyed the kind hospitality of the Fields Institute, partially supported by NSF grant DMS-0753152.

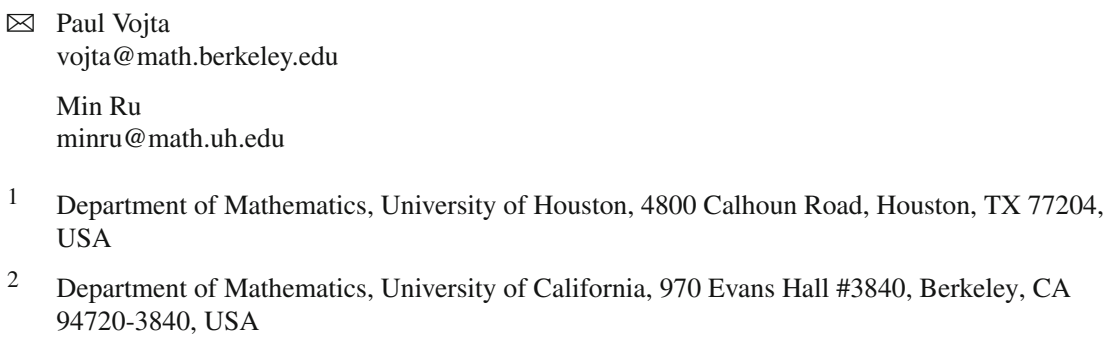




\section{Introduction}

Let $X$ be a projective variety and let $D$ be an effective Cartier divisor on $X$. Let $\mathscr{L}$ be a line sheaf on $X$. Other notations are as described in Sect. 5 and in our previous paper [16].

In [16], the authors introduced the birational Nevanlinna constant $\operatorname{Nev}_{\text {bir }}(\mathscr{L}, D)$, which extends the notion of the Nevanlinna constant $\operatorname{Nev}(\mathscr{L}, D)$ introduced by the first-named author [15], and proved the following results.

Theorem A Let $k$ be a number field, and let $S$ be a finite set of places of $k$ containing all archimedean places. Let $X$ be a projective variety over $k$, let $D$ be an effective Cartier divisor on $X$, and let $\mathscr{L}$ be a line sheaf on $X$. Then, for every $\epsilon>0$, there is a proper Zariski-closed subset $Z$ of $X$ such that the inequality

$$
m_{S}(x, D) \leq\left(\operatorname{Nev}_{\text {bir }}(\mathscr{L}, D)+\epsilon\right) h_{\mathscr{L}}(x)
$$

holds for all $x \in X(k)$ outside of $Z$.

Theorem B Let $X$ be a complex projective variety, let $D$ be an effective Cartier divisor, and let $\mathscr{L}$ be a line sheaf on $X$. Let $f: \mathbb{C} \rightarrow X$ be a holomorphic mapping with Zariski-dense image. Then, for every $\epsilon>0$,

$$
m_{f}(r, D) \leq \operatorname{exc}\left(\operatorname{Nev}_{\operatorname{bir}}(\mathscr{L}, D)+\epsilon\right) T_{f, \mathscr{L}}(r)
$$

Recall that $\operatorname{Nev}_{\text {bir }}(\mathscr{L}, D)$ and $\operatorname{Nev}(\mathscr{L}, D)$ are defined as follows. (In [16] we defined them only for $\mathbb{Q}$-Cartier divisors and required $\mu$ to be rational, but we are working over $\mathbb{R}$ in this paper. See Sect. 2 for more details on $\mathbb{R}$-Cartier divisors.)

In these definitions (and the rest of the paper), if $s$ is a nonzero global section (or nonzero rational section) of a line sheaf $\mathscr{L}$ on a variety, then $\operatorname{div}(s)$ is the corresponding Cartier divisor (so that $\mathscr{O}(\operatorname{div}(s)) \cong \mathscr{L}$ ), and if $\mathcal{B}=\left\{s_{1}, \ldots, s_{n}\right\}$ is a finite collection of nonzero global (or rational) sections, then $\operatorname{div}(\mathcal{B})=\sum \operatorname{div}\left(s_{i}\right)$.

Definition 1.1 Let $X$ be a complete variety, let $D$ be an effective $\mathbb{R}$-Cartier divisor on $X$, and let $\mathscr{L}$ be a line sheaf on $X$. Then we define

$$
\operatorname{Nev}_{\text {bir }}(\mathscr{L}, D)=\inf _{N, V, \mu} \frac{\operatorname{dim} V}{\mu},
$$

where the infimum passes over all triples $(N, V, \mu)$ such that $N \in \mathbb{Z}_{>0}, V$ is a linear subspace of $H^{0}\left(X, \mathscr{L}^{N}\right)$ with $\operatorname{dim} V>1$, and $\mu \in \mathbb{R}_{>0}$, with the following property. There exist a variety $Y$ and a proper birational morphism $\phi: Y \rightarrow X$ such that the following condition holds. For all $Q \in Y$ there is a basis $\mathcal{B}$ of $V$ such that

$$
\phi^{*} \operatorname{div}(\mathcal{B}) \geq \mu N \phi^{*} D
$$

in a Zariski-open neighborhood $U$ of $Q$, relative to the cone of effective $\mathbb{R}$-Cartier divisors on $U$. If there are no such triples $(N, V, \mu)$, then $\operatorname{Nev}_{\text {bir }}(\mathscr{L}, D)$ is defined to be $+\infty$. 
If $L$ is a Cartier divisor or Cartier divisor class on $X$, then we define $\operatorname{Nev}_{\text {bir }}(L, D)=$ $\operatorname{Nev}_{\text {bir }}(\mathscr{O}(L), D)$. We also define $\operatorname{Nev}_{\text {bir }}(D)=\operatorname{Nev}_{\text {bir }}(D, D)$.

The Nevanlinna constant $\operatorname{Nev}(\mathscr{L}, D)$ is defined similarly but without taking the birational model (i.e., it requires $Y$ to be the normalization of $X$ ). Obviously, $\operatorname{Nev}_{\text {bir }}(\mathscr{L}, D) \leq \operatorname{Nev}(\mathscr{L}, D)$.

The purpose of this note is to introduce the following variant of the Nevanlinna constant, based on a theorem of Evertse and Ferretti [3].

Definition 1.2 Let $X$ be a complete variety, let $D$ be an effective $\mathbb{R}$-Cartier divisor on $X$, and let $\mathscr{L}$ be a line sheaf on $X$. Then we define

$$
\operatorname{Nev}_{E F}(\mathscr{L}, D)=\inf _{N, \mu} \frac{\operatorname{dim} X+1}{\mu},
$$

where the infimum passes over all pairs $(N, \mu)$ such that $N \in \mathbb{Z}_{>0}$ and $\mu \in \mathbb{R}_{>0}$, with the following property. There exist a variety $Y$ and a proper birational morphism $\phi: Y \rightarrow X$ such that the following condition holds. For all $Q \in Y$ there exist a basepoint-free linear subspace $V \subseteq H^{0}\left(X, \mathscr{L}^{N}\right)$ with $\operatorname{dim} V=\operatorname{dim} X+1$ and a basis $\mathcal{B}$ of $V$ such that

$$
\phi^{*} \operatorname{div}(\mathcal{B}) \geq \mu N \phi^{*} D
$$

in a Zariski-open neighborhood $U$ of $Q$, relative to the cone of effective $\mathbb{R}$-divisors on $U$. If there are no such pairs $(N, \mu)$, then $\operatorname{Nev}_{\mathrm{EF}}(\mathscr{L}, D)$ is defined to be $+\infty$.

If $L$ is a Cartier divisor or Cartier divisor class on $X$, then we define $\operatorname{Nev}_{\mathrm{EF}}(L, D)=$ $\operatorname{Nev}_{E F}(\mathscr{O}(L), D)$. We also define $\operatorname{Nev}_{E F}(D)=\operatorname{Nev}_{E F}(D, D)$.

Our main result is as follows.

Main Theorem Let X be a geometrically integral variety over a number field $k$, let $D$ be an effective $\mathbb{R}$-Cartier divisor on $X$, and let $\mathscr{L}$ be a line sheaf on $X$. Then

$$
\operatorname{Nev}_{\text {bir }}(\mathscr{L}, D) \leq \operatorname{Nev} E F(\mathscr{L}, D)
$$

Combining this with Theorem A and Lemma 5.1, we then have

Theorem 1.3 Let $k$ be a number field, and let $S$ be a finite set of places of $k$ containing all archimedean places. Let $X$ be a projective variety over $k$, let $D$ be an effective $\mathbb{R}$-Cartier divisor on $X$, and let $\mathscr{L}$ be a line sheaf on $X$. Then, for every $\epsilon>0$, there is a proper Zariski-closed subset $Z$ of $X$ such that the inequality

$$
m_{S}(x, D) \leq\left(\operatorname{Nev}_{\mathrm{EF}}(\mathscr{L}, D)+\epsilon\right) h_{\mathscr{L}}(x)
$$

holds for all $x \in X(k)$ outside of $Z$.

These are special cases of Theorems 5.5 and 5.6, respectively. 
Corollary 1.4 Let $k$ be a number field, and let $S$ be a finite set of places of $k$ containing all archimedean places. Let $X$ be a projective variety over $k$, and let $D$ be an ample Cartier divisor on $X$. If $\operatorname{Nev}_{\mathrm{EF}}(D)<1$ then there is a proper Zariski-closed subset $Z$ of $X$ such that any set of $(S, D)$-integral points on $X$ has only finitely many points outside of $Z$.

Results similar to the above two theorems and the corollary are also true in the case of holomorphic curves.

Example 1.5 The inequality in the Main Theorem may be strict. For example, let $k$ be either a number field or the field of complex numbers, let $X$ be the blowing-up of $\mathbb{P}_{k}^{2}=\operatorname{Proj} k[x, y, z]$ at the point $P=[0: 0: 1]$, let $\pi X \rightarrow \mathbb{P}_{k}^{2}$ be the blowing-up morphism, let $E$ be the exceptional divisor, and let $\mathscr{L}=\pi^{*} \mathscr{O}(1) \otimes \mathscr{O}(E)$. Then $\mathscr{L}$ has degree -1 along $E$, so all global sections of $\mathscr{L}^{N}$ must vanish on $E$ for all $N>0$. In particular, $\mathscr{L}^{N}$ has base points for all $N>0$, so the same is true for all $V \subseteq H^{0}\left(X, \mathscr{L}^{N}\right)$ for all $N$. Therefore $\operatorname{Nev}_{\mathrm{EF}}(\mathscr{L}, D)=+\infty$ for all divisors $D$ on $X$.

On the other hand, let $\mathcal{B}=\left\{\pi^{*} x, \pi^{*} y, \pi^{*} z\right\} \subseteq H^{0}(X, \mathscr{L})$, let $V \subseteq H^{0}(X, \mathscr{L})$ be the subspace that it generates (this is actually all of $H^{0}(X, \mathscr{L})$ ), and let $D=\operatorname{div}\left(\pi^{*} z\right)$. Then, taking $N=1$ and $\mu=1$ in Definition 1 .1, we have $\operatorname{Nev}_{\text {bir }}(\mathscr{L}, D) \leq 1$. In particular,

$$
\operatorname{Nev}_{\text {bir }}(\mathscr{L}, D)<\operatorname{Nev}_{\mathrm{EF}}(\mathscr{L}, D)
$$

Theorem 1.3 implies a special case of the original theorem of Evertse and Ferretti, in which the collection of hypersurfaces is independent of the places $v \in S$ and the explicit bounds on the exceptional subset are omitted. Recovering the fuller EvertseFerretti theorem (allowing the collection of hypersurfaces to depend on $v$, but still omitting the bounds on the exceptional set) is possible, using a "multidivisor EvertseFerretti Nevanlinna constant"; we show how to recover the latter Evertse-Ferretti theorem from Theorem 5.6, which is a multidivisor version of Theorem 1.3 (see Theorem 6.1).

Basically, the multidivisor versions of the various Nevanlinna constants and related theorems allow one to prove theorems of Second Main Theorem type with "multidivisor proximity functions" in place of classical proximity functions. For example, in the number field case, if $D_{1}, \ldots, D_{q}$ are effective divisors on a projective variety $X$ and $\lambda_{D_{1}}, \ldots, \lambda_{D_{q}}$ are corresponding Weil functions, then one can define a multidivisor proximity function

$$
m_{S}\left(x, D_{1}, \ldots, D_{q}\right)=\frac{1}{[k: \mathbb{Q}]} \sum_{v \in S} \max _{1 \leq i \leq q} \lambda_{D_{i}, v}(x)
$$

for points $x \in X(k) \backslash \bigcup_{i}$ Supp $D_{i}$ (see Definition 4.5). Second Main Theorems of this type were introduced by the second-named author [19]; see also work of the first-named author [13].

Some other applications are also given. This includes discussion of a 1999 class of examples obtained by Faltings [4]. 
These examples consist of irreducible divisors $D$ on $\mathbb{P}^{2}$ for which $\mathbb{P}^{2} \backslash D$ has only finitely many integral points over any number ring, and over any localization of such a ring away from finitely many places.

Faltings' paper is notable for two reasons. First, the divisor $D$ is irreducible. Prior to the paper, the only divisors $D$ on $\mathbb{P}^{2}$ for which such statements were known were divisors with at least four irreducible components. The second reason is that (as noted at the very end of [4]) the paper gives examples of varieties for which finiteness of integral points is known, yet which cannot be embedded into semiabelian varieties. Prior to the paper, the only varieties for which such finiteness statements were known, and which could not be embedded into semiabelian varieties, were moduli spaces of abelian varieties.

Faltings' construction was further explored by Zannier [21] using methods of Zannier and Corvaja. Zannier considered a different family of examples, which has substantial overlap with the examples of Faltings but does not contain all of his examples. After that, Levin [11, §13] derived a generalization, using his method of large divisors, that encompasses the examples of both Faltings and Zannier.

The method of Faltings' paper is to apply the original "filtration method" of Faltings and Wüstholz [5], together with their probabilistic version of Schmidt's Subspace Theorem, to certain coherent ideal sheaves on a certain étale cover of $\mathbb{P}^{2} \backslash D$.

This use of ideal sheaves naturally suggested the use of "generalized Weil functions" of the second-named author [20,§7], which, as we noted in [16], are now more aptly called $b$-Weil functions. We first rephrased Faltings' proof in terms of $b$-Weil functions and replaced the work of Faltings-Wüstholz with the method of Evertse and Ferretti [3].

In an effort to express this modified proof using the Nevanlinna constant of the first-named author, we formulated the "birational Nevanlinna constant" $\mathrm{Nev}_{\text {bir }}(\mathscr{L}, D)$ (Definition 1.1). We found in [16] that this definition worked well for adapting Autissier's proof, but that it was not possible to apply it to Faltings' examples without involving the Evertse-Ferretti method. This was what led to the formulation of the "Evertse-Ferretti Nevanlinna constant" $\operatorname{Nev}_{\mathrm{EF}}(\mathscr{L}, D)$ (Definition 1.2).

The outline of this paper is as follows. Section 2 introduces $\mathbb{R}$-Cartier b-divisors on a complete variety $X$ and develops some of their basic properties. Section 3 recalls the concepts of $\mu$-growth and $\mu$-b-growth from [15] and [16, Def. 4.12], respectively, and gives a corresponding definition of $\mu$-EF-growth for the Evertse-Ferretti Nevanlinna constant. Section 4 defines "multi-divisor" counterparts to the Nevanlinna constants of Definitions 1.1 and 1.2 , as well as the original Nevanlinna constant $\operatorname{Nev}(\mathscr{L}, D)$. It also proves counterparts to the Main Theorems regarding the original and birational Nevanlinna constants, including Theorems A and B. Section 5 gives more information on the Evertse-Ferretti Nevanlinna constant, including some equivalent formulations of it, and proves the Main Theorem. Section 6 contains some brief applications of Theorem 1.3, including the earlier result of Evertse and Ferretti. Section 7 concludes this note by discussing a 1999 class of examples obtained by Faltings [4]. 


\section{$2 \mathbb{R}$-Cartier b-divisors and their $\mathbf{b}$-Weil functions}

This section introduces some notation for divisors and b-divisors with coefficients in $\mathbb{R}$. (We already used divisors with real coefficients in [16], but did not fully describe them there because we added them as a late change to the paper.)

Let $k$ be a field of characteristic zero. As in [16], a variety over $k$ is an integral scheme, separated and of finite type over $k$. It is said to be complete if it is proper over $k$.

\section{1 $\mathbb{Q}$-Cartier and $\mathbb{R}$-Cartier divisors}

If $X$ is a variety over $k$, then we let $\operatorname{CDiv}(X)$ denote the group of Cartier divisors on $X$. As in Lazarsfeld [10, 1.3.B], an $\mathbb{R}$-Cartier divisor on $X$ is an element of the group

$$
\operatorname{CDiv}_{\mathbb{R}}(X):=\operatorname{CDiv}(X) \otimes_{\mathbb{Z}} \mathbb{R}
$$

Thus, every $\mathbb{R}$-Cartier divisor $D$ on $X$ occurs as a finite sum $D=\sum c_{i} D_{i}$, with $c_{i} \in \mathbb{R}$ and $D_{i} \in \operatorname{CDiv}(X)$ for all $i$. An $\mathbb{R}$-Cartier divisor $D$ is effective if it can be written in the above form with $c_{i} \geq 0$ and $D_{i}$ effective for all $i$.

If $X$ is normal, then $\operatorname{CDiv}(X)$ is canonically identified with a subgroup of the group $\operatorname{Div}(X)$ of Weil divisors on $X$, and by Hartshorne [6, II 6.3A] a Cartier divisor on $X$ is effective if and only if it is effective as a Weil divisor. $\operatorname{Defining} \operatorname{CDiv}_{\mathbb{Q}}(X), \operatorname{Div}_{\mathbb{R}}(X)$, and $\operatorname{Div}_{\mathbb{Q}}(X)$ similarly to $(7)$, we have that the map $\operatorname{Div}(X) \rightarrow \operatorname{CDiv}(X)$ induces natural maps $\operatorname{Div}_{\mathbb{Q}}(X) \rightarrow \operatorname{CDiv}_{\mathbb{Q}}(X)$ and $\operatorname{Div}_{\mathbb{R}}(X) \rightarrow \operatorname{CDiv}_{\mathbb{R}}(X)$. These too are injective $(\mathbb{Q}$ is a localization of $\mathbb{Z}$, hence is flat over $\mathbb{Z}$, and $\mathbb{R}$ is flat over the field $\mathbb{Q}$, respectively). Moreover these maps again preserve the respective cones of effective divisors.

Since the maps $\operatorname{Div}(X) \rightarrow \operatorname{Div}_{\mathbb{Q}}(X) \rightarrow \operatorname{Div}_{\mathbb{R}}(X)$ are injective and respect the notion of effectivity, the maps $\operatorname{CDiv}(X) \rightarrow \operatorname{CDiv}_{\mathbb{Q}}(X) \rightarrow \operatorname{CDiv}_{\mathbb{R}}(X)$ have these same properties when $X$ is normal.

If $X$ is not normal, then an effective (integral) Cartier divisor on $X$ remains effective in $\operatorname{CDiv}_{\mathbb{Q}}(X)$ and $\operatorname{CDiv}_{\mathbb{R}}(X)$, but the converse does not hold. For example, the Cartier divisor $(y / x)$ on the cuspidal cubic curve $y^{2}=x^{3}$ is not effective, but it is effective as an $\mathbb{Q}$-Cartier or $\mathbb{R}$-Cartier divisor.

\subsection{B-divisors with coefficients in $\mathbb{Z}, \mathbb{Q}$, and $\mathbb{R}$}

B-divisors consider divisors not only on a given variety, but also on blowings-up of the variety. The prefix 'b' means birational.

Let $X$ be a variety. Following Boucksom et al. [1], a model over $X$ is a proper birational morphism $\pi: X_{\pi} \rightarrow X$, modulo isomorphism over $X$. This will be denoted $X_{\pi}$. The Zariski-Riemann space of $X$ is defined as

$$
\mathfrak{X}=\lim _{\pi} X_{\pi},
$$


and a Cartier b-divisor on a variety $X$ is an element of the group

$$
\operatorname{CDiv}(\mathfrak{X}):=\underset{\pi}{\lim } \operatorname{CDiv}\left(X_{\pi}\right) .
$$

Thus, each Cartier b-divisor on $X$ comes from a Cartier divisor $D$ on some model $X_{\pi}$. A Cartier b-divisor is said to be effective if $X_{\pi}$ and $D$ can be chosen such that $D$ is effective.

An $\mathbb{R}$-Cartier b-divisor on $X$ is an element of the group

$$
\begin{aligned}
\operatorname{CDiv}_{\mathbb{R}}(\mathfrak{X}): & =\operatorname{CDiv}(\mathfrak{X}) \otimes \mathbb{R} \\
& \cong \underset{\pi}{\lim } \operatorname{CDiv}_{\mathbb{R}}\left(X_{\pi}\right)
\end{aligned}
$$

An $\mathbb{R}$-Cartier b-divisor on $X$ is said to be effective if it comes from an effective $\mathbb{R}$ Cartier divisor on some model $X_{\pi}$. The group $\operatorname{CDiv}_{\mathbb{Q}}(\mathfrak{X})$ of $\mathbb{Q}$-Cartier b-divisors on $X$ is defined similarly.

Assume briefly that the variety $X$ is normal. By [16, Remark 2.3], a Cartier divisor on $X$ is effective if and only if the corresponding Cartier b-divisor on $X$ is effective. Therefore the map $\operatorname{CDiv}(X) \rightarrow \operatorname{CDiv}(\mathfrak{X})$ (from Cartier divisors on $X$ to Cartier bdivisors on $X$ ) is injective and preserves effectivity. Likewise, the maps $\operatorname{CDiv}(\mathfrak{X}) \rightarrow$ $\operatorname{CDiv}_{\mathbb{Q}}(\mathfrak{X}) \rightarrow \operatorname{CDiv}_{\mathbb{R}}(\mathfrak{X})$ are injective and preserve effectivity.

If $X$ is not normal, then an effective Cartier divisor on $X$ still remains effective as a Cartier b-divisor (or as a $\mathbb{Q}$-Cartier or $\mathbb{R}$-Cartier b-divisor), but not conversely. The latter is illustrated by the same example as before.

In contrast to (non-birational) Cartier divisors, though, a Cartier b-divisor on an arbitrary variety $X$ is effective if and only if it is effective as a $\mathbb{Q}$-Cartier or $\mathbb{R}$-Cartier b-divisor. This is because we can replace $X$ with its normalization without affecting any of these groups of b-divisors.

In [16, Prop. 4.10a], we showed that the group $\mathrm{CDiv}(\mathfrak{X})$ is a lattice-ordered group; i.e., an ordered group in which every two elements $\mathbf{D}_{1}$ and $\mathbf{D}_{2}$ have a least upper bound $\mathbf{D}_{1} \vee \mathbf{D}_{2}$. This, in turn, relied on [16, Lemma 4.8], which stated that if $D$ is a Cartier divisor on a variety $Y$, then there is a proper model $\pi: Y^{\prime} \rightarrow Y$ such that $\pi^{*} D$ is a difference of effective Cartier divisors with disjoint supports. This lemma remains true for $\mathbb{Q}$-Cartier divisors (by multiplying the divisor in question by a positive integer so as to clear denominators), but is no longer true for $\mathbb{R}$-Cartier divisors. An example of the latter is the $\mathbb{R}$-divisor $\alpha L_{1}-\beta L_{2}$, where $L_{1}$ and $L_{2}$ are distinct lines on $\mathbb{P}^{2}$ and $\alpha, \beta>0$ are real numbers such that $\alpha / \beta$ is irrational.

Therefore $\operatorname{CDiv}(\mathfrak{X})_{\mathbb{Q}}$ is a lattice-ordered group, but $\operatorname{CDiv}(\mathfrak{X})_{\mathbb{R}}$ is not. As it turns out, though, this is not an issue here. In this paper, the only instances of taking the least upper bound or greatest lower bound of two b-divisors involve only integral Cartier b-divisors. 


\subsection{B-Weil functions for $\mathbb{R}$-Cartier b-divisors}

Now assume that $k$ is either a number field or the field $\mathbb{C}$, and that $X$ is a complete variety over $k$. Then a b-Weil function for a $\mathbb{R}$-Cartier b-divisor on $X$ can be defined by $\mathbb{R}$-linearity, using the definition of $b$-Weil function for (integral) Cartier b-divisors, and these $b$-Weil functions can be used as usual to define proximity and counting functions.

Moreover, Ru and Vojta [16, Prop. 2.4 and Prop. 4.6] still hold in this context (i.e., b-Weil functions for $\mathbb{R}$-Cartier b-divisors have the same linearity, existence, and uniqueness properties; an $\mathbb{R}$-Cartier b-divisor is effective if and only if some associated b-Weil function is bounded from below by an $M_{k}$-constant; and if $U$ is a nonempty Zariski-open subset of a complete variety over a number field or over $\mathbb{C}$, then any function $\lambda: U(M) \rightarrow \mathbb{R}$ extends to a b-Weil function for at most one $\mathbb{R}$-Cartier b-divisor on $X$ ).

In addition, $\mathrm{Ru}$ and Vojta [16, Lemma 2.5] extends to $\mathbb{R}$-Cartier divisors:

Lemma 2.1 Let $X$ be a complete variety, and let $U_{1}, \ldots, U_{n}$ be Zariski-open subsets of $X$ that cover $X$. Let $D_{1}, \ldots, D_{n}$ be $\mathbb{R}$-Cartier divisors on $X$ such that $\left.D_{i}\right|_{U_{i}}$ is effective for all $i$, and let $\lambda_{D_{i}}$ be Weil functions for $D_{i}$ for all $i$. Then there is an $M_{k}$-constant $\gamma=\left(\gamma_{v}\right)$ such that, for all $v$ and all $x \in X\left(\bar{k}_{v}\right)$ there is an $i$ such that $x \in U_{i}$ and $\lambda_{D_{i}, v}(x) \geq \gamma_{v}$.

Proof By taking a finite refinement of $\left\{U_{i}\right\}$, we may assume that each $U_{i}$ is affine, and that $\left.D_{i}\right|_{U_{i}}=\sum_{j=1}^{n_{i}} c_{i j}\left(f_{i j}\right)$ for all $i$, where $f_{i j} \in \mathscr{O}\left(U_{i}\right) \backslash\{0\}$ and $c_{i j} \in \mathbb{R}_{>0}$ for all $i$ and $j$.

Then $\lambda_{D_{i}}$ is locally $M_{k}$-bounded from below on $U_{i}\left(M_{k}\right)$ for all $i$. Indeed, by Lang [9, Ch. 10, Prop. 1.3], the function $-\sum_{j} c_{j} \log \left\|f_{i j}\right\|$ is locally $M_{k}$-bounded from below on $U_{i}\left(M_{k}\right)$ for all $i$; therefore so is $\lambda_{D_{i}}$ since by definition of Weil function and Néron function the difference between the two functions is locally $M_{k}$-bounded.

The proof then concludes as in [16] (using standard properties of $M_{k}$-bounded sets).

Then [16, Prop. 3.3] extends to $\mathbb{R}$-Cartier b-divisors as follows.

Proposition 2.2 Let $X$ be a complete variety over a number field, let $D$ be an effective $\mathbb{R}$-Cartier divisor on $X$, let $\mathscr{L}$ be a line sheaf on $X$, let $V$ be a linear subspace of $H^{0}(X, \mathscr{L})$ with $\operatorname{dim} V>1$, and let $\mu \in \mathbb{R}_{>0}$.

Consider the following conditions.

(i) There exist a model $\pi: X_{\pi} \rightarrow X$ of $X$ such that for all $Q \in X_{\pi}$ there is a basis $\mathcal{B}$ of $V$ such that

$$
\pi^{*} \operatorname{div}(\mathcal{B}) \geq \mu \pi^{*} D
$$

in a Zariski-open neighborhood $U$ of $Q$, relative to the cone of effective $\mathbb{R}$-Cartier divisors on $U$.

(ii) There are finitely many bases $\mathcal{B}_{1}, \ldots, \mathcal{B}_{\ell}$ of $V$; Weil functions $\lambda_{\mathcal{B}_{1}}, \ldots, \lambda_{\mathcal{B}_{\ell}}$ for $\operatorname{div}\left(\mathcal{B}_{1}\right), \ldots, \operatorname{div}\left(\mathcal{B}_{\ell}\right)$, respectively; a Weil function $\lambda_{D}$ for $D ;$ and an $M_{k}$-constant c such that 


$$
\max _{1 \leq i \leq \ell} \lambda_{\mathcal{B}_{i}} \geq \mu \lambda \lambda_{D}-c
$$

(as functions $X\left(M_{k}\right) \rightarrow \mathbb{R} \cup\{+\infty\}$ ).

Then (i) implies (ii).

The proof is the same as in [16], except that Lemma 2.1 replaces [16, Lemma 2.5] and the step of multiplying by a positive integer $n$ is omitted.

\section{Growth conditions}

We first recall the definition of $\mu$-b-growth in [16] which appeared in the definition of $\operatorname{Nev}_{\text {bir }}(L, D)$. This can now be stated using $\mathbb{R}$-Cartier divisors instead of $\mathbb{Q}$-Cartier divisors.

Definition 3.1 ([16, Def. 4.12]) Let $X$ be a complete variety, let $D$ be an effective $\mathbb{R}$-Cartier divisor on $X$, let $\mathscr{L}$ be a line sheaf on $X$, let $V$ be a linear subspace of $H^{0}(X, \mathscr{L})$ with $\operatorname{dim} V>1$, and let $\mu>0$ be a real number. We say that $D$ has $\mu$-b-growth with respect to $V$ and $\mathscr{L}$ if there is a model $\phi: Y \rightarrow X$ of $X$ such that for all $Q \in Y$ there is a basis $\mathcal{B}$ of $V$ such that

$$
\phi^{*} \operatorname{div}(\mathcal{B}) \geq \mu \phi^{*} D
$$

in a Zariski-open neighborhood $U$ of $Q$, relative to the cone of effective $\mathbb{R}$-divisors on $U$. Also, we say that $D$ has $\mu$-b-growth with respect to $V$ if it satisfies the above condition with $\mathscr{L}=\mathscr{O}(D)$.

Remark 3.2 Basically, $\operatorname{Nev}_{\text {bir }}(\mathscr{L}, D)$, according to its definition, is the infimum of $(\operatorname{dim} V) / \mu$ over all triples $(N, V, \mu)$ such that $\mu>0$ and $N D$ has $\mu$-b-growth with respect to $V$ and $\mathscr{L}^{N}$.

Remark 3.3 Def. 4.12 of [16] requires $X$ to be normal, but this is unnecessary since the definition is birational in nature.

Remark 3.4 If an effective $\mathbb{R}$-Cartier divisor $D$ has $\mu$-b-growth with respect to $V$ and $\mathscr{L}$, then it also has $\mu^{\prime}$-b-growth with respect to $V$ and $\mathscr{L}$ for all $\mu^{\prime}<\mu$. Therefore, if $D$ is $\mathbb{Q}$-Cartier (or Cartier), then the value of $\operatorname{Nev}_{\text {bir }}(\mathscr{L}, D)$ according to Definition 1.1 coincides with the value according to [16, Def. 4.12], because one can require $\mu$ to be rational in Definition 1.1 without changing the value of $\operatorname{Nev}_{\text {bir }}(\mathscr{L}, D)$.

Here is an equivalent condition for $\mu$-b-growth that will be needed for the proof of Proposition 5.4, which compares $\mu$-b-growth with $\mu$-EF-growth. This condition is a special case of [16, Prop. 4.13]. For a fuller list of equivalent conditions, see loc. cit.

Proposition 3.5 Let $X$ be a complete variety, let $D$ be an effective $\mathbb{R}$-Cartier divisor on $X$, let $\mathscr{L}$ be a line sheaf on $X$, let $V$ be a linear subspace of $H^{0}(X, \mathscr{L})$ with $\operatorname{dim} V>1$, and let $\mu>0$ be a real number. Then $D$ has $\mu$-b-growth with respect to $V$ and $\mathscr{L}$ if and only if the following condition is true. 
For each place $v \in M_{k}$ there are finitely many bases $\mathcal{B}_{1}, \ldots, \mathcal{B}_{\ell}$ of $V$; local Weil functions $\lambda_{\mathcal{B}_{1}, v}, \ldots, \lambda_{\mathcal{B}_{\ell}, v}$ for $\operatorname{div}\left(\mathcal{B}_{1}\right), \ldots, \operatorname{div}\left(\mathcal{B}_{\ell}\right)$, respectively, at $v$; a local Weil function $\lambda_{D, v}$ for $D$ at $v$; and a constant $c$ such that

$$
\max _{1 \leq i \leq \ell} \lambda_{\mathcal{B}_{i}, v} \geq \mu \lambda \lambda_{D, v}-c
$$

(as functions $X\left(\bar{k}_{v}\right) \rightarrow \mathbb{R} \cup\{+\infty\}$ ).

We similarly rephrase the definition of the Evertse-Ferretti Nevanlinna constant $\operatorname{Nev}_{\mathrm{EF}}(\mathscr{L}, D)$ in terms of a definition similar that of $\mu$-b-growth, and is suitable for applying the work of Evertse and Ferretti [3].

Definition 3.6 Let $X$ be a complete variety, let $D$ be an effective $\mathbb{R}$-Cartier divisor on $X$, let $\mathscr{L}$ be a line sheaf on $X$, and let $\mu>0$ be a real number. We say that $D$ has $\mu$-EF-growth with respect to $\mathscr{L}$ if there is a model $\phi: Y \rightarrow X$ of $X$ such that for all $P \in Y$ there exist a base-point-free linear subspace $V \subseteq H^{0}(X, \mathscr{L})$ with $\operatorname{dim} V=\operatorname{dim} X+1$ and a basis $\mathcal{B}$ of $V$ such that

$$
\phi^{*} \operatorname{div}(\mathcal{B}) \geq \mu \phi^{*} D
$$

in a Zariski-open neighborhood $U$ of $P$, relative to the cone of effective $\mathbb{R}$-divisors on $U$. Also, we say that $D$ has $\mu$-EF-growth if it satisfies the above condition with $\mathscr{L}=\mathscr{O}(D)$.

Similarly to what was done with $\mu$-b-growth, we have the following proposition.

Proposition 3.7 Let $\mathscr{L}$ and $D$ be as in Definition 3.6. Then the Evertse-Ferretti Nevanlinna constant $\operatorname{Nev}_{\mathrm{EF}}(\mathscr{L}, D)$ is the infimum of $(\operatorname{dim} X+1) / \mu$ over all pairs $(N, \mu)$ such that $\mu>0$ is real and ND has $\mu$-EF-growth with respect to $\mathscr{L}^{N}$.

Remark 3.8 Let $\mathscr{L}$ and $D$ be as in Definition 3.6, and let $\mu$ and $r$ be positive real numbers. Then $r D$ has $\mu$-EF-growth with respect to $\mathscr{L}$ if and only if $D$ has $r \mu$-EFgrowth with respect to $\mathscr{L}$, and

$$
\operatorname{Nev}_{\mathrm{EF}}(\mathscr{L}, r D)=r \operatorname{Nev}_{\mathrm{EF}}(\mathscr{L}, D)
$$

In regard to the definition of $\mu$-EF-growth, we have the following equivalent statements (for notations, see our earlier paper [16]). These parallel the full list of [16, Prop. 4.13].

Proposition 3.9 Let $X$ be a complete variety, let $D$ be an effective $\mathbb{R}$-Cartier divisor on $X$, let $\mathscr{L}$ be a line sheaf on $X$, and let $\mu>0$ be a real number. Then the following are equivalent.

(i) D has $\mu$-EF-growth with respect to $\mathscr{L}$.

(ii) There are base-point-free linear subspaces $V_{1}, \ldots, V_{\ell}$ of $H^{0}(X, \mathscr{L})$, all of dimension $\operatorname{dim} X+1$, and corresponding bases $\mathcal{B}_{1}, \ldots, \mathcal{B}_{\ell}$ of $V_{1}, \ldots, V_{\ell}$, respectively, such that 


$$
\bigvee_{i=1}^{\ell} \operatorname{div}\left(\mathcal{B}_{i}\right) \geq \mu D
$$

relative to the cone of effective $\mathbb{R}$-Cartier $b$-divisors.

(iii) There are base-point-free linear subspaces $V_{1}, \ldots, V_{\ell}$ of $H^{0}(X, \mathscr{L})$, all of dimension $\operatorname{dim} X+1$; bases $\mathcal{B}_{1}, \ldots, \mathcal{B}_{\ell}$ of $V_{1}, \ldots, V_{\ell}$, respectively; Weil functions $\lambda_{\mathcal{B}_{1}}, \ldots, \lambda_{\mathcal{B}_{\ell}}$ for $\operatorname{div}\left(\mathcal{B}_{1}\right), \ldots, \operatorname{div}\left(\mathcal{B}_{\ell}\right)$, respectively; a Weil function $\lambda_{D}$ for $D$; and an $M_{k}$-constant $c$ such that

$$
\max _{1 \leq i \leq \ell} \lambda_{\mathcal{B}_{i}} \geq \mu \lambda_{D}-c
$$

(as functions $X\left(M_{k}\right) \rightarrow \mathbb{R} \cup\{+\infty\}$ ).

(iv) For each $v \in M_{k}$ there are base-point-free linear subspaces $V_{1}, \ldots, V_{\ell}$ of $H^{0}(X, \mathscr{L})$, all of dimension $\operatorname{dim} X+1$; bases $\mathcal{B}_{1}, \ldots, \mathcal{B}_{\ell}$ of $V_{1}, \ldots, V_{\ell}$, respectively; local Weil functions $\lambda_{\mathcal{B}_{1}, v}, \ldots, \lambda_{\mathcal{B}_{\ell}, v}$ at $v$ for $\operatorname{div}\left(\mathcal{B}_{1}\right), \ldots, \operatorname{div}\left(\mathcal{B}_{\ell}\right)$, respectively; a local Weil function $\lambda_{D, v}$ for $D$ at $v$; and a constant $c$ such that

$$
\max _{1 \leq i \leq \ell} \lambda_{\mathcal{B}_{i}, v} \geq \mu \lambda_{D, v}-c
$$

(as functions $X\left(\bar{k}_{v}\right) \rightarrow \mathbb{R} \cup\{+\infty\}$ ).

(v) The condition of (iv) holds for at least one place $v$.

Note that the join in (13) exists, because it involves only integral Cartier b-divisors.

Proof Conditions (ii) and (iii) are equivalent by [16, Prop. 4.10], and (iii)-(v) are equivalent by [16, Prop. 2.4]. The implication (ii) $\Longrightarrow$ (i) follows from [16, Lemma 4.14], with $D_{i}=\operatorname{div}\left(\mathcal{B}_{i}\right)$ for all $i$. (Note that, in the latter lemma, one can allow $\mu$ to be real and $D$ to be an $\mathbb{R}$-Cartier divisor: when working with $\mathbb{R}$-divisors in the proof, the step of multiplying by a positive integer $n$ is unnecessary; also, the last few lines in the proof work equally well with $\mathbb{R}$-Cartier divisors in place of $\mathbb{Q}$-Cartier divisors.)

To finish the proof, it will suffice to show that (i) implies (ii).

Assume that condition (i) is true. Let $\phi: Y \rightarrow X$ be a model that satisfies the condition of Definition 3.6. By quasi-compactness of $Y$, we may assume that only finitely many triples $(U, V, \mathcal{B})$ occur. Let $\left(U_{1}, V_{1}, \mathcal{B}_{1}\right), \ldots,\left(U_{\ell}, V_{\ell}, \mathcal{B}_{\ell}\right)$ be those triples. We may assume that $\bigvee \operatorname{div}\left(\mathcal{B}_{i}\right)$ is represented by a Cartier divisor $E$ on $Y$. Then, for each $i$,

$$
\left.E\right|_{U_{i}} \geq\left.\phi^{*} \operatorname{div}\left(\mathcal{B}_{i}\right)\right|_{U_{i}} \geq\left.\mu \phi^{*} D\right|_{U_{i}}
$$

relative to the cone of effective $\mathbb{R}$-Cartier divisors on $U_{i}$. This gives (13).

\section{Multidivisor Nevanlinna constants}

We can generalize the definitions of $\operatorname{Nev}(\mathscr{L}, D), \operatorname{Nev}_{\text {bir }}(\mathscr{L}, D)$, and $\operatorname{Nev}_{\mathrm{EF}}(\mathscr{L}, D)$ to incorporate multiple divisors in place of $D$, as follows. The main properties of the 
previously defined Nevanlinna constants extend to multidivisor Nevanlinna constants in a straightforward way.

\subsection{Definitions of multidivisor Nevanlinna constants}

Definition 4.1 Let $X$ be a complete variety, let $D_{1}, \ldots, D_{q}(q>0)$ be effective $\mathbb{R}$ Cartier divisors on $X$, and let $\mathscr{L}$ be a line sheaf on $X$. Then we make the following definitions. In each case the infimum of the empty set is taken to be $+\infty$.

(a).

$$
\operatorname{Nev}_{\text {bir }}\left(\mathscr{L}, D_{1}, \ldots, D_{q}\right)=\inf _{N, V, \mu} \frac{\operatorname{dim} V}{\mu},
$$

where the infimum passes over all triples $(N, V, \mu)$ such that $N \in \mathbb{Z}_{>0}, V$ is a linear subspace of $H^{0}\left(X, \mathscr{L}^{N}\right)$ with $\operatorname{dim} V>1$, and $\mu \in \mathbb{R}_{>0}$, with the following property. There exist a variety $Y$ and a proper birational morphism $\phi: Y \rightarrow X$ such that the following condition holds. For all $Q \in Y$ and all $i=1, \ldots, q$ there is a basis $\mathcal{B}$ of $V$ such that

$$
\phi^{*} \operatorname{div}(\mathcal{B}) \geq \mu N \phi^{*} D_{i}
$$

in a Zariski-open neighborhood $U$ of $Q$, relative to the cone of effective $\mathbb{R}$-Cartier divisors on $U$.

(b). $\operatorname{Nev}\left(\mathscr{L}, D_{1}, \ldots, D_{q}\right)$ is defined similarly, except that $\phi: Y \rightarrow X$ is only allowed to be the normalization map of $X$.

(c).

$$
\operatorname{Nev}_{\mathrm{EF}}\left(\mathscr{L}, D_{1}, \ldots, D_{q}\right)=\inf _{N, \mu} \frac{\operatorname{dim} X+1}{\mu}
$$

where the infimum passes over all pairs $(N, \mu)$ such that $N \in \mathbb{Z}_{>0}$ and $\mu \in$ $\mathbb{R}_{>0}$, with the following property. There exist a variety $Y$ and a proper birational morphism $\phi: Y \rightarrow X$ such that the following condition holds. For all $Q \in Y$ and all $i=1, \ldots, q$ there exist a base-point-free linear subspace $V \subseteq H^{0}\left(X, \mathscr{L}^{N}\right)$ with $\operatorname{dim} V=\operatorname{dim} X+1$, and a basis $\mathcal{B}$ of $V$, such that

$$
\phi^{*} \operatorname{div}(\mathcal{B}) \geq \mu N \phi^{*} D_{i}
$$

in a Zariski-open neighborhood $U$ of $Q$, relative to the cone of effective $\mathbb{R}$-divisors on $U$.

If $L$ is a Cartier divisor or Cartier divisor class on $X$, then we define $\operatorname{Nev}_{\text {bir }}(L$, $\left.D_{1}, \ldots, D_{q}\right)=\operatorname{Nev}_{\text {bir }}\left(\mathscr{O}(L), D_{1}, \ldots, D_{q}\right)$, and define $\operatorname{Nev}\left(L, D_{1}, \ldots, D_{q}\right)$ and $\operatorname{Nev}_{\mathrm{EF}}\left(L, D_{1}, \ldots, D_{q}\right)$ similarly.

Note that the above definitions reduce to the earlier ones when $q=1$, so the notation is not in conflict. 
Remark 4.2 These Nevanlinna constants can be described instead using the related concepts of $\mu$-growth as follows.

(a). As in Remark 3.2, $\operatorname{Nev}_{\text {bir }}\left(\mathscr{L}, D_{1}, \ldots, D_{q}\right)$ is the infimum of $(\operatorname{dim} V) / \mu$ over all triples $(N, V, \mu)$ such that $\mu>0$ and $N D_{i}$ has $\mu$-b-growth with respect to $V$ and $\mathscr{L}^{N}$ for all $i$.

(b). Similarly, $\operatorname{Nev}\left(\mathscr{L}, D_{1}, \ldots, D_{q}\right)$ can be described in the same way, but with $\mu$ growth instead of $\mu$-b-growth.

(c). Finally, as in Proposition 3.7, $\operatorname{Nev}_{\mathrm{EF}}\left(\mathscr{L}, D_{1}, \ldots, D_{q}\right)$ is the infimum of $(\operatorname{dim} X+$ 1) / $\mu$ over all pairs $(N, \mu)$ such that $\mu>0$ and $N D_{i}$ has $\mu$-EF-growth with respect to $\mathscr{L}^{N}$ for all $i$.

In parts (a) and (b), the subspace $V$ must be independent of $i$, but this is not the case in (c), so we actually have

$$
\operatorname{Nev}_{\mathrm{EF}}\left(\mathscr{L}, D_{1}, \ldots, D_{q}\right)=\min _{1 \leq i \leq q} \operatorname{Nev} \mathrm{EF}\left(\mathscr{L}, D_{i}\right)
$$

The inequality $\operatorname{Nev}_{\text {bir }}(\mathscr{L}, D) \leq \operatorname{Nev}(\mathscr{L}, D)$ carries over easily to the multidivisor case.

Proposition 4.3 Let $X, D_{1}, \ldots, D_{q}$, and $\mathscr{L}$ be as in Definition 4.1. Then

$$
\operatorname{Nev}_{\text {bir }}\left(\mathscr{L}, D_{1}, \ldots, D_{q}\right) \leq \operatorname{Nev}\left(\mathscr{L}, D_{1}, \ldots, D_{q}\right)
$$

Proof This inequality holds for the same reason as in our earlier paper [16, Lemma 3.1]: the infimum in the definition of $\operatorname{Nev}\left(\mathscr{L}, D_{1}, \ldots, D_{q}\right)$ is taken over a subset of the triples in the corresponding infimum for $\mathrm{Nev}_{\mathrm{bir}}$.

\subsection{Multidivisor Theorems A and B, and their equivalents for the multidivisor original Nevanlinna constant}

The Main Theorem (Theorems A and B) for the birational Nevanlinna constant, as well as its counterpart for the original (non-birational) Nevanlinna constant, carry over to the multidivisor case in the obvious way (and also to the case of $\mathbb{R}$-divisors), as follows.

Theorem 4.4 (Multidivisor Theorem A, and its non-birational equivalent) Let $k$ be a number field, and let $S$ be a finite set of places of $k$ containing all archimedean places. Let $X$ be a projective variety over $k$, let $D_{1}, \ldots, D_{q}(q>0)$ be effective $\mathbb{R}$-Cartier divisors on $X$, and let $\mathscr{L}$ be a line sheaf on $X$. For all $i=1, \ldots, q$ let $\lambda_{D_{i}}$ be a Weil function for $D_{i}$. Then, for every $\epsilon>0$, there are proper Zariski-closed subsets $Z$ and $Z_{\mathrm{bir}}$ of $X$ such that the inequalities

$$
\frac{1}{[k: \mathbb{Q}]} \sum_{v \in S} \max _{1 \leq i \leq q} \lambda_{D_{i}, v}(x) \leq\left(\operatorname{Nev}\left(\mathscr{L}, D_{1}, \ldots, D_{q}\right)+\epsilon\right) h_{\mathscr{L}}(x)
$$


and

$$
\frac{1}{[k: \mathbb{Q}]} \sum_{v \in S} \max _{1 \leq i \leq q} \lambda_{D_{i}, v}(x) \leq\left(\operatorname{Nev}_{\mathrm{bir}}\left(\mathscr{L}, D_{1}, \ldots, D_{q}\right)+\epsilon\right) h_{\mathscr{L}}(x)
$$

hold for all $x \in X(k)$ outside of $Z$ and $Z_{\mathrm{bir}}$, respectively.

The left-hand sides of (20) and (21) can be written more conveniently using the following definition.

Definition 4.5 Let $k, S, X, D_{1}, \ldots, D_{q}, \mathscr{L}$, and $\lambda_{D_{1}}, \ldots, \lambda_{D_{q}}$ be as in Theorem 4.4. Then the multidivisor proximity function for $D_{1}, \ldots, D_{q}$ is

$$
m_{S}\left(x, D_{1}, \ldots, D_{q}\right)=\frac{1}{[k: \mathbb{Q}]} \sum_{v \in S} \max _{1 \leq i \leq q} \lambda_{D_{i}, v}(x)
$$

for all $x \in X(k) \backslash \bigcup \operatorname{Supp} D_{i}$. This proximity function depends on the choices of the Weil functions, but the dependence is only up to $O(1)$. (One can also extend this definition to handle algebraic points $x \in X(\bar{k}) \backslash \bigcup \operatorname{Supp} D_{i}$.)

Then, for example, (21) can be written as

$$
m_{S}\left(x, D_{1}, \ldots, D_{q}\right) \leq\left(\operatorname{Nev}_{\text {bir }}\left(\mathscr{L}, D_{1}, \ldots, D_{q}\right)+\epsilon\right) h_{\mathscr{L}}(x) .
$$

Remark 4.6 We expect that conjectured Second Main Theorems should also hold with multidivisor proximity functions in place of proximity functions. An additional conjecture for this case is not necessary, though: conjectured Second Main Theorems for ordinary proximity functions already imply the corresponding conjectured Second Main Theorems with multidivisor proximity functions. Indeed, let $D_{1}, \ldots, D_{q}$ be reduced normal crossings divisors on a smooth projective variety $X$ over a number field $k$ or over $\mathbb{C}$. Let $\phi X^{\prime} \rightarrow X$ be an embedded resolution of $\bigcup$ Supp $D_{i}$, and let $D^{\prime}$ be a reduced divisor on $X^{\prime}$ (necessarily with normal crossings) whose support equals $\phi^{-1}\left(\bigcup \operatorname{Supp} D_{i}\right)$. Then a conjectured Second Main Theorem for $D^{\prime}$ on $X^{\prime}$ would imply the corresponding conjectural Second Main Theorem with multidivisor proximity function for $D_{1}, \ldots, D_{q}$ on $X$. The details are left to the reader.

One could also define a multidivisor counting function, but it would probably not be useful.

Proof of Theorem 4.4 We start with (21). This proof will follow [15], as summarized at the end of [16, Section 5].

Fix $\epsilon>0$.

By Remark 4.2a, there is a triple $(N, V, \mu)$ such that $\mu>0$,

$$
\frac{\operatorname{dim} V}{\mu}<\operatorname{Nev}_{\text {bir }}\left(\mathscr{L}, D_{1}, \ldots, D_{q}\right)+\epsilon
$$

and $N D_{i}$ has $\mu$-b-growth with respect to $V$ and $\mathscr{L}^{N}$ for all $i$. The latter means that, for all $i$, there exist a variety $Y_{i}$ and a proper birational morphism $\phi_{i}: Y_{i} \rightarrow X$ 
such that, for all $Q \in Y_{i}$, there is a basis $\mathcal{B}$ of $V \operatorname{such}$ that $\phi_{i}^{*} \operatorname{div}(\mathcal{B})-\mu N \phi_{i}^{*} D_{i}$ is an effective $\mathbb{R}$-divisor in a Zariski-open neighborhood of $Q$. By choosing a proper birational morphism $\phi: Y \rightarrow X$ that factors through all of the $\phi_{i}$, we may assume that $\phi_{i}=\phi$ for all $i$. Then, by compactness, there are finitely many bases $\mathcal{B}_{1}, \ldots, \mathcal{B}_{\ell}$ of $V$ such that, for all $i$ and all $Q \in Y$ there exists $j$ such that the $\mathbb{R}$-divisor $\phi^{*} \operatorname{div}\left(\mathcal{B}_{j}\right)-$ $\mu N \phi^{*} D_{i}$ is effective in a neighborhood of $Q$.

Choose Weil functions $\lambda_{\mathcal{B}_{j}}$ for $\operatorname{div}\left(\mathcal{B}_{j}\right)$ for all $j$ and $\lambda_{D_{i}}$ for $D_{i}$ for all $i$. Then, for all $v \in S$ and all $i=1, \ldots, q$ there is a constant $c_{v, i}$ such that

$$
\max _{1 \leq j \leq \ell} \lambda_{\mathcal{B}_{j}, v}(x) \geq \mu \lambda_{D_{i}, v}(x)-c_{v, i}
$$

for all $x \in Y(k) \backslash \bigcup \operatorname{Supp} D_{i}$. Therefore, up to constants,

$$
\mu \cdot m_{S}\left(x, D_{1}, \ldots, D_{q}\right) \leq \frac{1}{[k: \mathbb{Q}]} \sum_{v \in S} \max _{1 \leq j \leq \ell} \lambda_{\mathcal{B}_{j}, v}(x)<(\operatorname{dim} V+\epsilon) h_{\mathscr{L}}(x)
$$

for all $x \in Y(k)$ outside of a proper Zariski-closed subset; here the second step follows from Schmidt's Subspace Theorem in the form of [16, Thm. 2.10].

Dividing (25) by $\mu$, combining it with (24), and adjusting $\epsilon$ then gives (21). (The additive constant can be eliminated by decreasing $\epsilon$ a bit more and imposing a lower bound on the height.)

The proof of (20) is similar: one only needs to change $\mathrm{Nev}_{\text {bir }}$ to Nev and set $\phi$ and all of the $\phi_{i}$ equal to the normalization map.

In the complex analytic case, one can again define a multidivisor proximity function, and we then have the following theorem.

Theorem 4.7 (Multidivisor Theorem B, and its non-birational equivalent) Let X be a complex projective variety, let $D_{1}, \ldots, D_{q}(q>0)$ be effective $\mathbb{R}$-Cartier divisors on $X$, and let $\mathscr{L}$ be a line sheaf on $X$. Let $f: \mathbb{C} \rightarrow X$ be a holomorphic mapping with Zariski-dense image. Then, for every $\epsilon>0$,

$$
m_{f}\left(r, D_{1}, \ldots, D_{q}\right) \leq \operatorname{exc}\left(\operatorname{Nev}\left(\mathscr{L}, D_{1}, \ldots, D_{q}\right)+\epsilon\right) T_{f, \mathscr{L}}(r)
$$

and

$$
m_{f}\left(r, D_{1}, \ldots, D_{q}\right) \leq \operatorname{exc}\left(\operatorname{Nev}_{\text {bir }}\left(\mathscr{L}, D_{1}, \ldots, D_{q}\right)+\epsilon\right) T_{f, \mathscr{L}}(r)
$$

The proof is similar to the proof in the number field case, and is omitted.

\section{The Evertse-Ferretti Nevanlinna constant $\operatorname{Nev}_{\mathrm{EF}}(\mathscr{L}, D)$}

This section provides more basic properties of the Evertse-Ferretti Nevanlinna constant (Definition 1.2), and proves the Main Theorem. 


\subsection{Mumford's theory of degree of contact}

The proof of the Main Theorem also relies on some results from Mumford's theory of degree of contact.

In this theory, we let $k$ be a field of characteristic 0 , let $x_{1}, \ldots, x_{q}$ be homogeneous coordinates on $\mathbb{P}_{k}^{q-1}$, let $Y \subseteq \mathbb{P}_{k}^{q-1}$ be a projective variety, let $H_{Y}(m)=$ $\operatorname{dim}_{k} H^{0}(Y, \mathscr{O}(m))$ be the Hilbert function of $Y$, let $I_{Y} \subseteq k\left[x_{1}, \ldots, x_{q}\right]$ be the ideal of $Y$, let $S(Y)=k\left[x_{1}, \ldots, x_{q}\right] / I_{Y}$ be the homogeneous coordinate ring of $Y$, and let $\mathbf{c}=\left(c_{1}, \ldots, c_{q}\right) \in \mathbb{R}_{\geq 0}^{q}$. Then (as is standard) for all $m \geq 0$ we define

$$
S_{Y}(m, \mathbf{c})=\max \left(\sum_{i=1}^{H_{Y}(m)} \mathbf{a}_{i} \cdot \mathbf{c}\right)
$$

where the maximum is taken over all sets of monomials $\left\{x^{\mathbf{a}_{1}}, \ldots, x^{\mathbf{a}_{H_{Y}(m)}}\right\}$ whose residue classes modulo $I_{Y}$ form a basis for $S(Y)_{m}=H^{0}(Y, \mathscr{O}(m))$.

In Mumford's theory it is better to consider only varieties that are geometrically integral, because $H^{0}(Y, \mathscr{L})$ is much harder to control when $Y$ becomes reducible upon base change to a larger field. Fortunately, though, theorems about Zariski-dense sets of rational points are all vacuously true on (integral) varieties that are not geometrically integral, because of the following simple fact.

Lemma 5.1 Let $Y$ be an (integral) variety over a field $k$ of characteristic 0 . If $Y(k)$ is Zariski dense, then $Y$ is geometrically integral.

Proof Since $Y_{\text {reg }}$ is open and Zariski dense, it contains a rational point. The existence of such a point implies that $Y$ is geometrically integral. Indeed, let $P$ be such a point, and let $k^{\prime}$ be the algebraic closure of $k$ in the function field $K(Y)$. Then $\mathscr{O}_{Y, P}$ is regular, hence normal. Since $k^{\prime}$ is integral over $k, \mathscr{O}_{Y, P}$ contains $k^{\prime}$. Therefore its residue field contains $k^{\prime}$, so $k=k^{\prime}$ is algebraically closed in $K(Y)$, and therefore $Y$ is geometrically integral.

Lemma 5.2 Let $k$ and $q$ be as above, let $Y \subseteq \mathbb{P}_{k}^{q-1}$ be a geometrically integral projective variety, and let $E$ be an extension field of $k$. Let $Y_{E}=Y \times_{k} E$, viewed as a projective variety in $\mathbb{P}_{E}^{q-1}$ via the map obtained from $Y \hookrightarrow \mathbb{P}_{k}^{q-1}$ by base change. Then, for all $\mathbf{c} \in \mathbb{R}_{\geq 0}^{q}$ and all $m \geq 0$,

$$
H_{Y_{E}}(m)=H_{Y}(m)
$$

and

$$
S_{Y_{E}}(m, \mathbf{c})=S_{Y}(m, \mathbf{c})
$$

Proof We have $H^{0}\left(Y_{E}, \mathscr{O}(m)\right)=H^{0}(Y, \mathscr{O}(m)) \otimes_{k} E$ (by cohomology and flat base change, for example). Therefore $H_{Y}(m)=H_{Y_{E}}(m)$ for all $m$. Also $S_{Y}(m, \mathbf{c})=$ $S_{Y_{E}}(m, \mathbf{c})$ because the computation is the same in both cases. 
Proposition 5.3 Let $k$ and $q$ be as above, let $Y \subseteq \mathbb{P}_{k}^{q-1}$ be a geometrically integral projective variety, and let $n=\operatorname{dim} Y$. Assume that $Y$ is not contained in any coordinate hyperplane of $\mathbb{P}^{q-1}$. Then the inequality

$$
c_{j_{0}}+\cdots+c_{j_{n}} \leq(n+1)\left(\frac{S_{Y}(m, \mathbf{c})}{m H_{Y}(m)}\right)(1+O(1 / m))
$$

holds for all $\mathbf{c} \in \mathbb{R}_{\geq 0}^{q}$ and all collections $j_{0}, \ldots, j_{n}$ of indices such that the linear system on $Y$ generated by $x_{j_{0}}, \ldots, x_{j_{n}}$ is base point free. Here the implicit constant in $O(1 / m)$ depends only on $Y$.

Proof By Lemma 5.2 we may assume that $k$ is algebraically closed.

Let $\mathbf{c}$ and $j_{0}, \ldots, j_{n}$ be as in the statement of Proposition 5.3, and let $m \in \mathbb{Z}_{>0}$. By the theory of degree of contact (see Ru [15, Lemma 3.2], which combines EvertseFerretti [2, Thm. 4.1] and Ru [14, Lemma 3.2]),

$$
c_{j_{0}}+\cdots+c_{j_{n}} \leq \frac{e_{Y}(\mathbf{c})}{\Delta} \leq \frac{n+1}{m}\left(\frac{S_{Y}(m, \mathbf{c})}{H_{Y}(m)}+(2 n+1) \Delta \max _{1 \leq j \leq q} c_{j}\right) .
$$

We claim that

$$
\max _{j} c_{j} \leq O\left(\frac{S_{Y}(m, \mathbf{c})}{m H_{Y}(m)}\right)
$$

with the implicit constant depending only on $Y$. Pick $j_{0}, \ldots, j_{n}$ such that that $c_{j_{0}}=\max _{j} c_{j}$ and $x_{j_{1}} / x_{j_{0}}, \ldots, x_{j_{n}} / x_{j_{0}}$ form a transcendence base for $K(Y)$ over $k$. Then, for each $m>0$, the monomials in $x_{j_{0}}, \ldots, x_{j_{n}}$ of total degree $m$ are linearly independent in $H^{0}(Y, \mathscr{O}(m))$, so the set $\left\{x_{j_{0}}^{\ell_{0}} \cdots x_{j_{n}}^{\ell_{n}}: \ell_{0}+\cdots+\ell_{n}=m\right\}$ of such monomials can be extended to a basis of $H^{0}(Y, \mathscr{O}(m))$. From the definition of $S_{Y}(m, \mathbf{c})$, and the fact that $H_{Y}(m)=\frac{\Delta}{n !} m^{n}+O\left(m^{n-1}\right)$, it then follows that

$$
\begin{aligned}
S_{Y}(m, \mathbf{c}) & \geq \sum_{\ell_{0}+\cdots+\ell_{n}=m} c_{j_{0}} \ell_{0} \\
& =\frac{c_{j_{0}} m}{n+1}\left(\begin{array}{c}
n+m \\
n
\end{array}\right) \\
& =\frac{c_{j_{0}} m}{n+1}\left(\frac{m^{n}}{n !}+O\left(m^{n-1}\right)\right) \\
& =\frac{c_{j_{0}}}{n+1}\left(\frac{1}{\Delta}+O\left(\frac{1}{m}\right)\right) m H_{Y}(m) .
\end{aligned}
$$

This proves (32). Combining (31) and (32) then gives (30). 


\subsection{Proof of the Main Theorem}

We are now ready to prove the Main Theorem. It will be proved in the multidivisor setting (which reduces to the Main Theorem when $q=1$ ) - see Theorem 5.5.

This proof, in turn, reduces to the following Proposition, which compares the notions of $\mu$-b-growth and $\mu$-EF-growth. Again, this is done in the multidivisor setting.

Proposition 5.4 Let $X$ be a geometrically integral variety over a number field $k$, let $D_{1}, \ldots, D_{q}(q>0)$ be effective $\mathbb{R}$-Cartier divisors on $X$, let $\mathscr{L}$ be a line sheaf on $X$, and let $\mu>0$ be a real number. Assume that $D_{i}$ has $\mu$-EF-growth with respect to $\mathscr{L}$ for all $i$. Then, for all $\epsilon>0$, there exist a positive integer $m$, a real number $\nu>0$, and a linear subspace $V \subseteq H^{0}\left(X, \mathscr{L}^{m}\right)$, such that

$$
\frac{\operatorname{dim} V}{v} \leq \frac{\operatorname{dim} X+1}{\mu}+\epsilon,
$$

and such that $m D_{i}$ has $v$-b-growth with respect to $V$ and $\mathscr{L}^{m}$ for all $i$.

Proof Assume that $D_{1}, \ldots, D_{q}$ all have $\mu$-EF-growth with respect to $\mathscr{L}$. Fix a place $v$ of $k$. By the implication (i) $\Longrightarrow$ (iv) of Proposition 3.9, there exist base-pointfree linear subspaces $V_{1}, \ldots, V_{\ell}$ of $H^{0}(X, \mathscr{L})$, all of dimension $\operatorname{dim} X+1$; bases $\mathcal{B}_{1}, \ldots, \mathcal{B}_{\ell}$ of $V_{1}, \ldots, V_{\ell}$, respectively; local Weil functions $\lambda_{\mathcal{B}_{1}, v}, \ldots, \lambda_{\mathcal{B}_{\ell}, v}$ at $v$ for $\operatorname{div}\left(\mathcal{B}_{1}\right), \ldots, \operatorname{div}\left(\mathcal{B}_{\ell}\right)$, respectively; local Weil functions $\lambda_{D_{i}, v}$ for $D_{i}$ at $v$ for all $i$; and a constant $c$ such that

$$
\max _{1 \leq j \leq \ell} \lambda_{\mathcal{B}_{j}, v} \geq \mu \lambda_{D_{i}, v}-c \quad \text { for all } i
$$

(as functions $\left.X\left(\bar{k}_{v}\right) \rightarrow \mathbb{R} \cup\{+\infty\}\right)$.

Let $\left\{s_{1}, \ldots, s_{p}\right\}$ be the elements of $\bigcup_{j} \mathcal{B}_{j}$. These sections determine a morphism $\Phi: X \rightarrow \mathbb{P}^{p-1}$. Let $Y$ be the image. Since the $s_{j}$ are nonzero sections, $Y$ is not contained in any coordinate hyperplane. Also note that $\Phi$ need not be a closed embedding; in fact, it may happen that $\operatorname{dim} Y<\operatorname{dim} X$. However, it is true that $Y$ is geometrically integral. Let $n=\operatorname{dim} Y$.

For each $j=1, \ldots, p$, choose a local Weil function $\lambda_{\left(s_{j}\right), v}$ for the divisor $\left(s_{j}\right)$ at $v$. Since each $\left(s_{j}\right)$ is effective, we may assume that each $\lambda_{\left(s_{j}\right), v}$ is nonnegative.

Fix $D \in\left\{D_{1}, \ldots, D_{q}\right\}$. Let $x \in X\left(\bar{k}_{v}\right)$ such that none of $s_{1}, \ldots, s_{p}$ vanish at $x$. We will apply (30) with $\mathbf{c}=\left(\lambda_{\left(s_{1}\right), v}(x), \ldots, \lambda_{\left(s_{p}\right), v}(x)\right)$. By (15), there is an index $j$ such that

$$
\lambda_{\mathcal{B}_{j}, v}(x) \geq \mu \lambda_{D, v}(x)+O(1)
$$

where the implicit constant does not depend on $x$ (or $j$ ). Write $\mathcal{B}_{j}=\left\{s_{j_{0}}, \ldots, s_{j_{n}}\right\}$. Then

$$
c_{j_{0}}+\cdots+c_{j_{n}}=\lambda_{\left(s_{j_{0}}\right), v}(x)+\cdots+\lambda_{\left(s_{j_{n}}\right), v}(x)=\lambda_{\mathcal{B}_{j}, v}(x)+O(1)
$$


where the implicit constant does not depend on $x$. Since there are only finitely many possible values for $j$, the constant may also be taken independent of $j$.

Combining (30), (36), and (35) gives

$$
\begin{aligned}
S_{Y}(m, \mathbf{c}) & \geq \frac{m H_{Y}(m)}{(n+1)(1+O(1 / m))} \mu \lambda_{D, v}(x)+O(1) \\
& =v m \lambda_{D, v}(x)+O(1),
\end{aligned}
$$

where again the implicit constant does not depend on $x$, and

$$
v=\frac{\mu H_{Y}(m)}{(n+1)(1+O(1 / m))} .
$$

Let $V \subseteq H^{0}\left(X, \mathscr{L}^{m}\right)$ be the pull-back of $H^{0}(Y, \mathscr{O}(m))$. Then $\operatorname{dim} V=H_{Y}(m)$. Since $\operatorname{dim} X \geq n$, we have

$$
\frac{\operatorname{dim} V}{v}=\frac{(n+1)(1+O(1 / m))}{\mu} \leq \frac{\operatorname{dim} X+1}{\mu}\left(1+O\left(\frac{1}{m}\right)\right) .
$$

Thus (33) holds for all sufficiently large $m$.

On the other hand, by the definition of $S_{Y}(m, \mathbf{c})$ and our choice of $\mathbf{c}$, there are bases $\mathcal{B}_{1}, \ldots, \mathcal{B}_{r}$ of $V$ and corresponding local Weil functions $\lambda_{\mathcal{B}_{1}, v}, \ldots, \lambda_{\mathcal{B}_{r}, v}$ such that

$$
S_{Y}(m, \mathbf{c})=\max _{1 \leq j \leq r} \lambda \mathcal{B}_{j}, v(\Phi(x))
$$

for all $x \in X\left(\bar{k}_{v}\right)$. Thus, after pulling the bases back to $V$ and the local Weil functions back to $X$, we see that $S_{Y}(m, \mathbf{c})$ equals the left-hand side of (11), and hence $m D$ has $\nu$-b-growth with respect to $V$ and $\mathscr{L}^{m}$.

Since this holds for all sufficiently large $m$, we may choose $m$ such that $m D_{i}$ has $\nu$-b-growth with respect to $V$ and $\mathscr{L}^{m}$ for all $i$ (note that $V$ depends only on $m$ ).

We now prove the following Multidivisor Main Theorem, which reduces to the earlier Main Theorem when $q=1$.

Theorem 5.5 (Multidivisor Main Theorem) Let $X$ be a geometrically integral variety over a number field $k$, let $D_{1}, \ldots, D_{q}(q>0)$ be effective $\mathbb{R}$-Cartier divisors on $X$, and let $\mathscr{L}$ be a line sheaf on $X$. Then

$$
\operatorname{Nev}_{\text {bir }}\left(\mathscr{L}, D_{1}, \ldots, D_{q}\right) \leq \operatorname{Nev}_{E F}\left(\mathscr{L}, D_{1}, \ldots, D_{q}\right)
$$

Proof We may assume that $\operatorname{Nev}_{\mathrm{EF}}\left(\mathscr{L}, D_{1}, \ldots, D_{q}\right)<\infty$ (otherwise there is nothing to prove).

Let $\epsilon>0$. By Remark 4.2c, there is a pair $(N, \mu)$, with $N \in \mathbb{Z}_{>0}$ and $\mu \in \mathbb{R}_{>0}$, such that $N D_{i}$ has $\mu$-EF-growth with respect to $\mathscr{L}^{N}$ for all $i$, and such that

$$
\frac{\operatorname{dim} X+1}{\mu}<\operatorname{Nev}_{\mathrm{EF}}\left(\mathscr{L}, D_{1}, \ldots, D_{q}\right)+\epsilon
$$


By Proposition 5.4, there exist a positive integer $m$, a real number $v>0$, and a linear subspace $V \subseteq H^{0}\left(X, \mathscr{L}^{m N}\right)$ such that

$$
\frac{\operatorname{dim} V}{v} \leq \frac{\operatorname{dim} X+1}{\mu}+\epsilon,
$$

and such that $m N D$ has $v$-b-growth with respect to $V$ and $\mathscr{L}^{m N}$. Then, by Remark 4.2a, (39), and (38),

$$
\operatorname{Nev}_{\text {bir }}\left(\mathscr{L}, D_{1}, \ldots, D_{q}\right) \leq \frac{\operatorname{dim} V}{v}<\operatorname{Nev}_{\mathrm{EF}}\left(\mathscr{L}, D_{1}, \ldots, D_{q}\right)+2 \epsilon
$$

and the proof concludes by letting $\epsilon$ go to zero.

Theorem 5.6 Let $k$ be a number field, and let $S$ be a finite set of places of $k$ containing all archimedean places. Let $X$ be a projective variety over $k$; let $D_{1}, \ldots, D_{q}$ be effective $\mathbb{R}$-Cartier divisors on $X$; and let $\mathscr{L}$ be a line sheaf on $X$. Then, for every $\epsilon>0$, there is a proper Zariski-closed subset $Z$ of $X$ such that the inequality

$$
m_{S}\left(x, D_{1}, \ldots, D_{q}\right) \leq\left(\operatorname{Nev}_{\mathrm{EF}}\left(\mathscr{L}, D_{1}, \ldots, D_{q}\right)+\epsilon\right) h_{\mathscr{L}}(x)
$$

holds for all $x \in X(k)$ outside of $Z$.

Proof By Lemma 5.1, we may assume that $X$ is geometrically integral. The theorem is then immediate from Definition 4.5, Theorem 4.4, and Theorem 5.5.

Versions of these theorems also hold for holomorphic curves. Their statements and proofs are entirely similar, and are left to the reader.

\section{Some applications}

In this section, we recover some known results by computing $\operatorname{Nev} \operatorname{EF}(\mathscr{L}, D)$ and then applying Theorem 1.3.

Let $X$ be a projective variety of dimension $n$ over a number field $k$ (the analytic case will be similar). Let $D_{1}, \ldots, D_{q}$ be effective Cartier divisors on $X$. In this section, such divisors will be said to be in general position if for all $I \subseteq\{1, \ldots, q\}$ with $|I| \leq n+1$, the intersection $\bigcap_{i \in I} \operatorname{Supp} D_{i}$ either is empty or has dimension $n-|I|$. A theorem of Evertse and Ferretti. We assume that $D_{1}, \ldots, D_{q}$ are in general position on $X$. In addition, we assume that there exist an ample divisor $A$ on $X$ and positive integers $d_{1}, \ldots, d_{q}$ such that $D_{i}$ is linearly equivalent to $d_{i} A$ for all $i=1, \ldots, q$. By replacing $D_{i}$ with $\left(d / d_{i}\right) D_{i}$, where $d=\operatorname{lcm}\left\{d_{1}, \ldots, d_{q}\right\}$, and $A$ with $d A$, we may assume that $d_{i}=1$ for all $i$. Let $D=D_{1}+\cdots+D_{q}$. To compute $\operatorname{Nev}_{\mathrm{EF}}(A, D)$, we take $N$ such that $N A$ is very ample, so there is a morphism $\phi: X \rightarrow \mathbb{P}^{m}$ and hyperplanes $H_{1}, \ldots, H_{q}$ in $\mathbb{P}^{m}$ such that $\phi^{*} H_{i}=N D_{i}$ for all $i$. Since $D_{1}, \ldots, D_{q}$ are in general position on $X$, for each point $P \in X$ there are at most $n=$ $\operatorname{dim} X$ divisors among $\left\{D_{1}, \ldots, D_{q}\right\}$ passing through $P$, so one may choose distinct 
$i_{0}, \ldots, i_{n} \in\{1, \ldots, q\}$ such that $D_{i_{0}}, \ldots, D_{i_{n}}$ includes all of the $D_{j}$ passing through $P$. Then $\phi^{*} H_{j_{i}}, i=0, \ldots, n$, (regarding $H$ as a section $\left.H \in H^{0}\left(\mathbb{P}^{m}, \mathscr{O}_{\mathbb{P} m}(1)\right)\right)$ forms a basis of a subspace $V \subset H^{0}(X, N A)$ with $\operatorname{dim} V=n+1$ (or, equivalently, the $\mathbb{Q}$-divisors $\frac{1}{N} \phi^{*} H_{j_{i}}, i=0, \ldots, n$, are actually integral divisors, and they define a basis of a subspace $V \subset H^{0}(X, A)$ with $\left.\operatorname{dim} V=n+1\right)$. By the condition on general position, this subspace is base point free. Furthermore, we have, for every irreducible component $E$ of $D$ with $P \in E$,

$$
\frac{1}{\operatorname{ord}_{E}(D)} \sum_{i=0}^{n} \operatorname{ord}_{E}\left(\frac{1}{N} \phi^{*} H_{j_{i}}\right) \geq 1
$$

Hence, from the definition,

$$
\operatorname{Nev}_{\mathrm{EF}}(D) \leq n+1
$$

Thus we recover the following important theorem of Evertse and Ferretti (without the explicit bounds on the exceptional set).

Theorem 6.1 (Evertse-Ferretti [3]) Let $k$ be a number field, let $S$ be a finite set of places of $k$, and let $X$ be a projective variety over $k$. Further, for each $v \in S$ let $D_{1}^{(v)}, \ldots, D_{q_{v}}^{(v)}$ be effective Cartier divisors on $X$ in general position. Assume that there exist an ample divisor $A$ on $X$ and positive integers $d_{v, i}$ such that $D_{i}^{(v)}$ is linearly equivalent to $d_{v, i} A$ for all $v \in S$ and all $i=1, \ldots, q_{v}$. Also, for each $v$ and $i$, let $\lambda_{D_{i}^{(v)}}$ be a Weil function for $D_{i}^{(v)}$. Then, for every $\epsilon>0$,

$$
\frac{1}{[k: \mathbb{Q}]} \sum_{v \in S} \sum_{i=1}^{q_{v}} \frac{1}{d_{v, i}} \lambda_{D_{i}^{(v)}, v}(x) \leq(\operatorname{dim} X+1+\epsilon) h_{A}(x)
$$

holds for all k-rational points outside a proper Zariski closed subset of X.

Proof Indeed, for each $v \in S$ let $D^{(v)}=D_{1}^{(v)}+\cdots+D_{q_{v}}^{(v)}$. Applying (41) to $D^{(v)}$ for all $v \in S$, we have

$$
\operatorname{Nev}_{\mathrm{EF}}\left(A,\left(D^{(v)}\right)_{v \in S}\right) \leq n+1
$$

by (18). This gives (42) by Theorem 5.6.

(In the special case in which the divisors $\left\{D_{i}^{(v)}: v \in S, 1 \leq i \leq q_{v}\right\}$ are in general position (ignoring duplicates with different $v$ ), it is possible to use Theorem 1.3 instead of Theorem 5.6.)

Seshadri constants and a theorem of Heier and Levin. We now only assume that $D_{1}, \ldots, D_{q}$ are in general position on $X$. Let $A$ be an ample Cartier divisor on $X$. Denote by $\epsilon_{D_{j}}(A)$ the Seshadri constant of $D_{j}$ with respect to $A$, which is defined as

$$
\epsilon_{D_{j}}(A)=\sup \left\{\gamma \in \mathbb{R}: \mathrm{A}-\gamma D_{j} \text { is nef }\right\} .
$$


Noticing that the statement in (43) involves $\epsilon$, it suffices to prove the inequality $\sum_{j=1}^{q} \sum_{v \in S} c_{j} \lambda_{D_{j}, v}(x)<(n+1+\epsilon) h_{A}(x)$ in place of (43), with rational $c_{j}$ close to $\epsilon_{D_{j}}(A)$ chosen such that $A-c_{j} D_{j}$ is ample for all $j$. By passing to $\mathbb{Q}$-Cartier divisors and replacing $D_{j}$ with $c_{j} D_{j}$ we can further assume that $c_{j}=1$ for all $j$.

In this case, we compute $\operatorname{Nev}_{\mathrm{EF}}(A, D)$ for $D=D_{1}+\cdots+D_{q}$. Take $N$ such that $q N A$ is very ample, $N D_{j}$ is integral for all $j$, and $N\left(A-D_{j}\right)$ is base point free for all $j$. According to Heier and Levin (see $[8, \S 3]$ ), there is a morphism $\phi: X \rightarrow \mathbb{P}^{m}$ and hyperplanes $H_{1}, \ldots, H_{q}$ in $\mathbb{P}^{m}$ such that $\phi^{*} H_{j} \geq N D_{j}$ for all $j$ and $\phi^{*} H_{1}, \ldots, \phi^{*} H_{q}$ are in general position. Then, for every $P \in X$, one can find hyperplanes $H_{j_{i}}$, $i=0, \ldots, n$, with $\left\{i_{0}, \ldots, i_{n}\right\} \subset\{1, \ldots, q\}$ such that the collection includes all $\phi^{*} H_{j}$ passing through $P, \phi^{*} H_{j_{i}} \geq N D_{j_{i}}$ for all $i$, and $\phi^{*} H_{j_{i}}, i=1, \ldots, n$, are in general position. Hence $\phi^{*} H_{j_{i}}, i=0, \ldots, n$, forms a basis of a base-point free subspace $V \subset H^{0}(X, N A)$ with $\operatorname{dim} V=n+1$. Furthermore, for every irreducible component $E$ of $D$ with $P \in E$, we have

$$
\frac{1}{\operatorname{ord}_{E}(D)} \sum_{i=0}^{n} \operatorname{ord}_{E}\left(\frac{1}{N} \phi^{*} H_{j_{i}}\right) \geq 1
$$

Thus

$$
\operatorname{Nev}_{\mathrm{EF}}(A, D) \leq n+1
$$

Then, by Theorem 1.3, we recover the following theorem.

Theorem 6.2 (Heier-Levin [8]) Let X be a projective variety of dimension $n$ defined over a number field $k$. Let $D_{1}, \ldots, D_{q}$ be effective Cartier divisors on $X$ in general position. Let $S$ be a finite set of places of $k$. Let $A$ be an ample Cartier divisor on $X$. Then, for $\epsilon>0$, there exists a proper Zariski-closed subset $Z \subset X$ such that for all points $x \in X(k) \backslash Z$,

$$
\sum_{j=1}^{q} \epsilon_{D_{j}}(A) m_{S}\left(x, D_{j}\right)<(n+1+\epsilon) h_{A}(x),
$$

where, for all $j, \epsilon_{D_{j}}(A)$ is the Seshadri constant of $D_{j}$ with respect to $A$.

This also works for subschemes in general position; see [8].

Divisors in l-subgeneral position and a theorem of $\mathrm{He}$ and $\mathrm{Ru}$. We only assume that $D_{1}, \ldots, D_{q}$ are in $l$-subgeneral position on $X$.

Recall the definition of $l$-subgeneral position: Let $V$ be a projective variety and $X \subset V$ be an irreducible subvariety of dimension $n$. Cartier divisors $D_{1}, \ldots, D_{q}$ on $V$ are said to be in $l$-subgeneral position on $X$ if for every choice $J \subset\{1, \ldots, q\}$ with $\# J \leq l+1$,

$$
\operatorname{dim}\left(\left(\bigcap_{j \in J} \operatorname{Supp} D_{j}\right) \cap X\right) \leq l-\# J
$$


The important tool to deal with $l$-subgeneral position is the following result, which is originally due to Quang (see Lemma 3.1 in [12]).

Lemma 6.3 ([12]) Let $k$ be a number field. Let $X \subset \mathbb{P}_{k}^{M}$ be a projective variety of dimension $n$. Let $H_{1}, \ldots, H_{l+1}$ be hyperplanes in $\mathbb{P}_{k}^{M}$ which are in l-subgeneral position on $X$ with $l \geq n$. Let $L_{1}, \ldots, L_{l+1}$ be the normalized linear forms defining $H_{1}, \ldots, H_{l+1}$ respectively. Then there exist linear forms $L_{1}^{\prime}, \ldots, L_{n+1}^{\prime}$ on $\mathbb{P}_{k}^{M}$ such that

(a) $L_{1}^{\prime}=L_{1}$.

(b) For every $t \in\{2, \ldots, n+1\}, L_{t}^{\prime} \in \operatorname{span}_{k}\left(L_{2}, \ldots, L_{l-n+t}\right)$; i.e., $L_{t}^{\prime}$ is a $k$-linear combination of $L_{2}, \ldots, L_{l-n+t}$.

(c) The hyperplanes defined by $L_{1}^{\prime}, \ldots, L_{n+1}^{\prime}$ are in general position on $X$.

Using this lemma, similar to the second case, we can prove that, under the assumptions that $\epsilon_{D_{j}}(A)=1$ for $j=1, \ldots, q$ and that $D_{1}, \ldots, D_{q}$ are in $l$-subgeneral position on $X$, for $D=D_{1}+\cdots+D_{q}$,

$$
\operatorname{Nev}_{\mathrm{EF}}(A, D) \leq(l-n+1)(n+1)
$$

Thus, we have

Theorem 6.4 (He-Ru [7]) Let $k$ be a number field and let $S$ be a finite set of places of $k$. Let $X$ be a projective variety of dimension $n$ over $k$. Let $D_{1}, \ldots, D_{q}$ be effective divisors on $X$ in $l$-subgeneral position with $l \geq n$. Let $A$ be an ample Cartier divisor on $X$. Then, for all $\epsilon>0$, there is a proper Zariski-closed subset $Z \subset X$ such that the inequality

$$
\sum_{j=1}^{q} \epsilon_{D_{j}}(A) m_{S}\left(x, D_{j}\right)<[(l-n+1)(n+1)+\epsilon] h_{A}(x)
$$

holds for all points $x \in X(k) \backslash Z$,

This also works for subschemes in $l$-subgeneral position, by blowing them up; see [7].

\section{An example of Faltings}

In this section we use the notion $\operatorname{Nev}_{\mathrm{EF}}(D)$ to recover the proof of a class of examples of Faltings that appeared in his Baker's Garden article [4]. Recall that these examples consist of irreducible divisors $D$ on $\mathbb{P}^{2}$ for which $\mathbb{P}^{2} \backslash D$ has only finitely many integral points over $\mathscr{O}_{k, S}$, where $\mathscr{O}_{k, S}$ is the localization of the ring of integers of a number field $k$ away from a finite set $S$ of places of $k$.

Note that none of the applications in Sect. 6 used the birational model in Definition 1.2. In this section, however, this model is essential.

The main result, Theorem 7.2, covers all of Faltings' examples, yet its proof follows rather directly from Corollary 1.4. Since the latter corollary relies on Schmidt's 
Subspace Theorem, it necessarily involves varieties that can be embedded into semiabelian varieties (actually $\mathbb{G}_{\mathrm{m}}^{N}$ ). Thus, Faltings' examples can be viewed as examples where one adds components to the divisor $D$ to obtain a divisor whose complement can be embedded into a semiabelian variety, in such a way that the resulting diophantine inequality is strong enough to give a useful inequality for the original divisor $D$.

In particular, the Shafarevich conjecture (on semistable abelian varieties over a given number field with good reduction outside of a given finite set of places, proved by Faltings in 1983) stands out as presently the only diophantine result with all the hallmarks of a result proved by Thue's method (ineffective, but with bounds on the number of counterexamples), but which has not been proved by Thue's method. This theorem amounts to showing finiteness of integral points on $\mathcal{A}_{g, n}$, which also has no embedding into a semiabelian variety. It would be interesting to know if some variant of the above approach could be used to derive finiteness of integral points on $\mathcal{A}_{g, n}$ (and therefore the Shafarevich conjecture) by methods that ultimately rely on Thue's method.

In this section, we give a revised proof of Faltings' result. We will split Faltings' main result into a geometric part and an arithmetic part. The geometric part (Theorem 7.1) guarantees that examples with certain properties exist, while the arithmetic part (Theorem 7.2) says that in each such example $\mathbb{P}^{2} \backslash D$ has only finitely many integral points over $\mathscr{O}_{k, S}$. We prove only the arithmetic part here, since that is the part that involves the Evertse-Ferretti Nevanlinna constant.

The first (geometric) part of Faltings' result is stated as follows.

Theorem 7.1 (Faltings) Let $k$ be a field of characteristic zero, and let X be a smooth geometrically irreducible algebraic surface over $k$. Then, for all sufficiently positive line sheaves $\mathscr{L}$ on $X$, there exists a morphism $f: X \rightarrow \mathbb{P}^{2}$ that satisfies the following conditions.

(i) $f^{*} \mathscr{O}(1) \cong \mathscr{L}$.

(ii) The ramification locus $Z$ of $f$ is smooth and irreducible, and the ramification index is 2.

(iii) The restriction of $f$ to $Z$ is birational onto its image $D \subseteq \mathbb{P}^{2}$.

(iv) $D$ is nonsingular except for cusps and simple double points.

(v) Let $Y \rightarrow X \rightarrow \mathbb{P}^{2}$ denote the Galois closure of $X \rightarrow \mathbb{P}^{2}$ (i.e., the normalization of $X$ in the Galois closure of $K(X)$ over $K\left(\mathbb{P}^{2}\right)$ ). Also let $n=\operatorname{deg} f$. Then $Y$ is smooth and its Galois group over $\mathbb{P}^{2}$ is the full symmetric group $S_{n}$.

(vi) The ramification locus of $Y$ over $\mathbb{P}^{2}$ is the sum of distinct conjugate effective divisors $Z_{i j}, 1 \leq i<j \leq n$. They have smooth supports, and are disjoint with the following two exceptions. Points of $Y$ lying over double points of $D$ are fixed points of a subgroup $S_{2} \times S_{2}$ of $S_{n}$, and they lie on $Z_{i j} \cap Z_{\ell m}$ with distinct indices $i, j, \ell, m$. Points of $Y$ lying over cusps of $D$ are fixed points of a subgroup $S_{3}$ of $S_{n}$, and lie on $Z_{i j} \cap Z_{i \ell} \cap Z_{j \ell}$.

For a proof of this theorem, and also an explicit description of the "sufficiently positive" condition on $\mathscr{L}$, see Faltings' paper [4]. 
For convenience, write $Z_{i j}=Z_{j i}$ when $i, j \in\{1, \ldots, n\}$ and $i>j$. Let

$$
A_{i}=\sum_{j: j \neq i} Z_{i j} \quad \text { and } \quad M=\sum A_{i}=\sum_{i \neq j} Z_{i j} .
$$

Let $L$ be the divisor class of $\mathscr{L}$ on $X$, and let it also denote the pull-back of this divisor class to $Y$. In addition, let $d=\operatorname{deg} D$. We then have

$$
2 \sum_{i<j} Z_{i j}=\sum A_{i}=M \sim d L
$$

Using the setup as above, we now use Evertse-Ferretti Nevanlinna constants together with Corollary 1.4 to prove the other part of Faltings' result.

Theorem 7.2 Let $k$ be a number field and let $S$ be a finite set of places of $k$. Let $Y, n$, $\left\{Z_{i j}\right\}_{i<j},\left\{A_{i}\right\}_{i}$, and $M$ be as in Theorem 7.1 and the discussion following it. Also let $\alpha$ be a rational number such that $M-\alpha A_{i}$ is an ample $\mathbb{Q}$-divisor for all $i$. Then:

(a). if $\alpha>6$ then no set of $\mathscr{O}_{k, S}$-integral points on $Y \backslash \bigcup Z_{i j}$ is Zariski-dense, and

(b). if $\alpha>8$ then every set of $\mathscr{O}_{k, S}$-integral points on $Y \backslash \bigcup Z_{i j}$ is finite.

Since $Y \backslash \cup Z_{i j}$ is an étale cover of $\mathbb{P}^{2} \backslash D$, the above conclusions also hold for $\mathbb{P}^{2} \backslash D$ (see Serre [17, §4.2] or Vojta [18, § 5.1]).

The first part of the proof of this theorem is the following proposition, which contains all of the geometry specific to the situation of Theorem 7.1.

Proposition 7.3 Let $k$ be a number field, and let $Y, n,\left\{Z_{i j}\right\}_{i<j},\left\{A_{i}\right\}_{i}, M$, and $\alpha$ be as in Theorem 7.2. Assume that $n \geq 4$. Fix Weil functions $\lambda_{i j}$ for each $Z_{i j}$. Let $\beta$ be an integer such that $\beta \alpha \in \mathbb{Z}$ and such that $\beta M$ and all $\beta\left(M-\alpha A_{i}\right)$ are very ample. Fix an embedding $Y \hookrightarrow \mathbb{P}_{k}^{N}$ associated to a complete linear system of $\beta M$, and regard $Y$ as a subvariety of $\mathbb{P}_{k}^{N}$ via this embedding. Then

(a). There exist a finite list $H_{1}, \ldots, H_{q}$ of hyperplanes in $\mathbb{P}_{k}^{N}$, with associated Weil functions $\lambda_{H_{j}}$ for all $j$, and constants $c_{v}$ for all $v \in M_{k}$, with the following property. Let $\mathscr{J}$ be the collection of all three-element subsets $J=\left\{j_{0}, j_{1}, j_{2}\right\}$ of $\{1, \ldots, q\}$ for which $Y \cap H_{j_{0}} \cap H_{j_{1}} \cap H_{j_{2}}=\emptyset$. Then $\mathscr{J} \neq \emptyset$, and the inequality

$$
\max _{J \in \mathscr{J}} \sum_{j \in J} \lambda_{H_{j}}(y) \geq \beta \alpha \sum_{i<j} \lambda_{i j}(y)-c_{v}
$$

holds for all $v \in M_{k}$ and all $y \in Y\left(\bar{k}_{v}\right)$ not lying on the support of any $Z_{i j}$ or on any of the $H_{j}$.

(b). Let $C$ be an integral curve in $Y$, not contained in the support of any $Z_{i j}$. Then there exist a finite list $H_{1}, \ldots, H_{q}$ of hyperplanes, with associated Weil functions as before, and constants $c_{v}$ for all $v \in M_{k}$, with the following property. Let $\mathscr{J}$ be the collection of all two-element subsets $J=\left\{j_{0}, j_{1}\right\}$ of $\{1, \ldots, q\}$ for which 
$C \cap H_{j_{0}} \cap H_{j_{1}}=\emptyset$. Then $\mathscr{J} \neq \emptyset$, and the inequality

$$
\max _{J \in \mathscr{J}} \sum_{j \in J} \lambda_{H_{j}}(y) \geq \frac{\beta \alpha}{2} \sum_{i<j} \lambda_{i j}(y)-c_{v}
$$

holds for all $v \in M_{k}$ and for all but finitely many $y \in C\left(\bar{k}_{v}\right)$.

The proof of this proposition, in turn, relies mainly on two lemmas. These lemmas replace Faltings' computations of ideals associated to indices.

Lemma 7.4 Let $i, j, \ell, m$ be distinct indices. Then:

(a). there exist hyperplanes $H_{0}, H_{1}$, and $H_{2}$ in $\mathbb{P}_{k}^{N}$, such that

$$
Y \cap H_{0} \cap H_{1} \cap H_{2}=\varnothing
$$

and

$$
\left.\left(H_{0}+H_{1}+H_{2}\right)\right|_{Y}-\beta \alpha\left(Z_{i j}+Z_{\ell m}\right)
$$

is an effective Cartier divisor on $Y$; and

(b). given any integral curve $C \subseteq Y$ not contained in any of the $Z_{a b}$, there are hyperplanes $H_{0}$ and $H_{1}$ in $\mathbb{P}_{k}^{N}$, such that $C \cap H_{0} \cap H_{1}=\varnothing$ and

$$
\left.\left(H_{0}+H_{1}\right)\right|_{C}-\left.Z_{i j}\right|_{C}
$$

is an effective Cartier divisor on $C$.

Proof Let $\sigma_{i}$ and $\sigma_{j}$ be the canonical sections of $\mathscr{O}\left(A_{i}\right)$ and $\mathscr{O}\left(A_{j}\right)$, respectively. Then the linear system

$$
\sigma_{i}^{\beta \alpha} \cdot \Gamma\left(Y, \beta\left(M-\alpha A_{i}\right)\right)+\sigma_{j}^{\beta \alpha} \cdot \Gamma\left(Y, \beta\left(M-\alpha A_{j}\right)\right)
$$

has base locus Supp $A_{i} \cap \operatorname{Supp} A_{j}$, since the first summand has base locus Supp $A_{i}$ and the second has base locus Supp $A_{j}$. This intersection consists of the union of $Z_{i j}$ and finitely many closed points. Choose an element of this linear system, sufficiently generic so that it does not vanish identically on any irreducible component of $Z_{\ell m}$, and let $H_{0}$ be the associated hyperplane in $\mathbb{P}_{k}^{N}$. Then $\left.H_{0}\right|_{Y}-\beta \alpha Z_{i j}$ is an effective divisor.

Similarly let $\sigma_{\ell}$ and $\sigma_{m}$ be the canonical sections of $\mathscr{O}\left(A_{\ell}\right)$ and $\mathscr{O}\left(A_{m}\right)$, respectively, and let $H_{1}$ be the hyperplane associated to an element of

$$
\sigma_{\ell}^{\beta \alpha} \cdot \Gamma\left(Y, \beta\left(M-\alpha A_{\ell}\right)\right)+\sigma_{m}^{\beta \alpha} \cdot \Gamma\left(Y, \beta\left(M-\alpha A_{m}\right)\right),
$$

chosen sufficiently generically such that $H_{1}$ does not contain any irreducible component of $H_{0} \cap Y$. Then $\left.H_{1}\right|_{Y}-\beta \alpha Z_{\ell m}$ is effective. 
By construction, $Y \cap H_{0} \cap H_{1}$ is a finite union of closed points, so we can let $H_{2}$ be a hyperplane that avoids those points to ensure that $Y \cap H_{0} \cap H_{1} \cap H_{2}=\emptyset$. By construction,

$$
\left(\left.H_{0}\right|_{Y}-\beta \alpha Z_{i j}\right)+\left(\left.H_{1}\right|_{Y}-\beta \alpha Z_{\ell m}\right)+\left.H_{2}\right|_{Y}
$$

is effective, and this is the divisor (46). This proves (a).

For part (b), let $\sigma_{i}$ be as above, and let $H_{0}$ be the hyperplane associated to an element of $\sigma_{i}^{\beta \alpha} \cdot \Gamma\left(Y, \beta\left(M-\alpha A_{i}\right)\right)$, chosen generically so that $H_{0}$ does not contain $C$. Let $H_{1}$ be a hyperplane in $\mathbb{P}_{k}^{N}$, chosen so that $C \cap H_{0} \cap H_{1}=\emptyset$. Since $\left.H_{0}\right|_{C}-\left.Z_{i j}\right|_{C}$ is an effective divisor, so is (47).

Lemma 7.5 Let $i, j, \ell$ be distinct indices. Then:

(a). there exist hyperplanes $H_{0}, H_{1}$, and $H_{2}$ in $\mathbb{P}_{k}^{N}$, such that

$$
Y \cap H_{0} \cap H_{1} \cap H_{2}=\varnothing
$$

and

$$
\left.\left(H_{0}+H_{1}+H_{2}\right)\right|_{Y}-\beta \alpha\left(Z_{i j}+Z_{i \ell}+Z_{j \ell}\right)
$$

is an effective Cartier divisor on $Y$; and

(b). given any integral curve $C \subseteq Y$ not contained in any of the $Z_{a b}$, there are hyperplanes $H_{0}$ and $H_{1}$ in $\mathbb{P}_{k}^{N}$, such that $C \cap H_{0} \cap H_{1}=\emptyset$ and

$$
\left.\left(H_{0}+H_{1}\right)\right|_{C}-\left.\beta \alpha\left(Z_{i j}+Z_{i \ell}\right)\right|_{C}
$$

is an effective Cartier divisor on $C$.

Proof Let $\sigma_{i}$ and $\sigma_{j}$ be as in the preceding proof. Choose a section of the linear system

$$
\sigma_{i}^{\beta \alpha} \cdot \Gamma\left(Y, \beta\left(M-\alpha A_{i}\right)\right)+\sigma_{j}^{\beta \alpha} \cdot \Gamma\left(Y, \beta\left(M-\alpha A_{j}\right)\right),
$$

and let $H_{0}$ be the associated hyperplane. Then $\left.H_{0}\right|_{Y}-\beta \alpha Z_{i j}$ is effective. We may assume that the choice of $H_{0}$ is sufficiently generic so that $H_{0}$ does not contain any irreducible component of $A_{\ell}$.

Next let $\sigma_{\ell}$ be the canonical section of $\mathscr{O}\left(A_{\ell}\right)$, and let $H_{1}$ be the hyperplane associated to a section of

$$
\sigma_{\ell}^{\beta \alpha} \cdot \Gamma\left(Y, \beta\left(M-\alpha A_{\ell}\right)\right)
$$

Then $\left.H_{1}\right|_{Y}-\beta \alpha\left(Z_{i \ell}+Z_{j \ell}\right)$ is effective. We may assume that $H_{1}$ does not contain any irreducible component of $Y \cap H_{0}$.

Again, $Y \cap H_{0} \cap H_{1}$ consists of finitely many points, and we choose $H_{2}$ to be any hyperplane not meeting any of these points. Part (a) then concludes as in the previous lemma. 
For part (b), let $H_{0}$ and $H_{1}$ be the hyperplanes associated to suitably chosen sections of $\sigma_{i}^{\beta \alpha} \cdot \Gamma\left(Y, \beta\left(M-\alpha A_{i}\right)\right)$ and $\Gamma(Y, \beta M)$, respectively. As in the previous lemma, we then have $C \cap H_{0} \cap H_{1}=\emptyset$. Since $\left.H_{0}\right|_{C}-\left.\beta \alpha\left(Z_{i j}+Z_{i \ell}\right)\right|_{C}$ is effective, so is (48).

Proof of Proposition 7.3 First consider part (a) of the proposition.

Fix a place $v \in M_{k}$. Apply Lemmas 7.4a and 7.5 a to all possible collections $i, j, \ell, m$ and $i, j, \ell$ of indices, respectively. This involves only finitely many applications, so only finitely many hyperplanes occur. Let $H_{1}, \ldots, H_{q}$ be those hyperplanes.

The conditions in Theorem 7.1 on the intersections of the divisors $Z_{i j}$ imply that there is a constant $C_{v}$ such that, for each $y \in Y(k)$ not in $\bigcup \operatorname{Supp} Z_{i j}$, one of the following conditions holds.

(i) $\lambda_{i j}(y) \leq C_{v}$ for all $i$ and $j$;

(ii) there are indices $i$ and $j$ such that $\lambda_{i j}(y)>C_{v}$ but $\lambda_{a b}(y) \leq C_{v}$ in all other cases;

(iii) there are distinct indices $i, j, \ell, m$ such that $\lambda_{i j}(y)>C_{v}$ and $\lambda_{\ell m}(y)>C_{v}$ but $\lambda_{a b}(y) \leq C_{v}$ in all other cases; or

(iv) there are indices $i, j, \ell$ such that $\max \left\{\lambda_{i j}(y), \lambda_{i \ell}(y), \lambda_{j \ell}(y)\right\}>C_{v}$, but $\lambda_{a b}(y) \leq C_{v}$ if $\{a, b\} \nsubseteq\{i, j, \ell\}$.

For case (iii), (44) follows from Lemma 7.4a, since one can take $J$ corresponding to the hyperplanes occurring in the lemma, and the inequality will then follow from effectivity of (46). Case (ii) follows as a special case of this lemma, since $n \geq 4$. Case (iv) follows from Lemma 7.5a, by a similar argument. Finally, in case (i) there is nothing to prove. This proves (a).

For part (b), let $H_{1}, \ldots, H_{q}$ be a finite collection of hyperplanes occurring in all possible applications of Lemmas $7.4 \mathrm{~b}$ and $7.5 \mathrm{~b}$ with the given curve $C$. We have cases (i)-(iv) as before. Cases (ii) and (iii) follow from Lemma 7.4b, where we may assume without loss of generality that $\lambda_{i j}(y) \geq \lambda_{\ell m}(y)$ to obtain (45) from effectivity of (47). Similarly, case (iv) follows from Lemma $7.5 \mathrm{~b}$ after a suitable permutation of the indices, and case (i) is again trivial.

Remark 7.6 It will not actually be needed in the sequel, but the collections $\left(c_{v}\right)$ of constants in each part of Proposition 7.3 are actually $M_{k}$-constants. This follows directly from our paper [16, Prop. 2.4].

Remark 7.7 It is possible (and, in fact, slightly easier) to write Proposition 7.3 in terms of Cartier b-divisors instead of Weil functions (for notations, see [16]). For example, one can replace (44) with

$$
\bigvee_{J \in \mathscr{J}} \sum_{j \in J} H_{j} \geq \beta \alpha \sum_{i<j} Z_{i j}
$$

relative to the cone of effective Cartier b-divisors.

In the proof, one would let $\phi: Y \rightarrow X$ be a model for which the left-hand side of (49) pulls back to an ordinary divisor, and for each $P \in X$ one would consider four cases: 
(i) $P \notin Z_{i j}$ for all $i, j$;

(ii) $P \in Z_{i j}$ for exactly one pair $i, j$;

(iii) there are distinct indices $i, j, \ell, m$ such that $P \in Z_{i j}$ and $P \in Z_{\ell m}$, but $P \notin Z_{a b}$ for all other components; and

(iv) there are indices $i, j, \ell$ such that $P$ lies on at least two of $Z_{i j}, Z_{i \ell}$, and $Z_{j \ell}$, but $P \notin Z_{a b}$ if $\{a, b\} \nsubseteq\{i, j, \ell\}$.

In each case let $U$ be the complement of all $Z_{a b}$ that do not contain $P$. Then $U$ is an open neighborhood of $P$ in $X$, and there is a set $J$ of indices such that $\sum_{j \in J} H_{j}-$ $\beta \alpha \sum_{a<b} Z_{a b}$ is effective on $U$. This set $J$ is obtained from Lemmas 7.4 or 7.5, as appropriate.

We chose to keep the phrasing in terms of Weil functions, however, since that is the phrasing that will be most convenient for the next step in the proof of Theorem 7.2.

The two parts of Proposition 7.3 say that $\beta \sum Z_{i j}=(\beta / 2) M$ has $\alpha$-EF-growth and $(\alpha / 2)$-EF-growth, respectively, with respect to $\mathscr{O}(\beta M)$, and therefore

$$
\operatorname{Nev}_{E F}\left(\beta M, \frac{\beta}{2} M\right) \leq \frac{3}{\alpha} \quad \text { and } \quad \operatorname{Nev}_{E F}\left(\beta M, \frac{\beta}{2} M\right) \leq \frac{4}{\alpha}
$$

respectively, on $Y$ and $C$, respectively. By Remark 3.8, these become

$$
\operatorname{Nev}_{E F}(\beta M) \leq \frac{6}{\alpha} \quad \text { and } \quad \operatorname{Nev}_{E F}(\beta M) \leq \frac{8}{\alpha}
$$

respectively.

Proof of Theorem 7.2(a). By (50) and the assumption $\alpha>6$, we have

$$
\operatorname{Nev}_{E F}(\beta M) \leq \frac{6}{\alpha}<1
$$

The result then follows by Corollary 1.4.

(b). Let $Z$ be the Zariski closure of a set of $D$-integral points on $Y$. By part (a), $Z \neq Y$, so it will suffice to show that no irreducible component of $Z$ can be a curve. This holds because, on any curve $C$ in $Y$ not contained in Supp $M$,

$$
\operatorname{Nev}_{\mathrm{EF}}(\beta M) \leq \frac{8}{\alpha}<1
$$

and we conclude as before.

Data Availibility Statement Data sharing is not applicable to this article as no datasets were generated or analysed during the current study. 
Open Access This article is licensed under a Creative Commons Attribution 4.0 International License, which permits use, sharing, adaptation, distribution and reproduction in any medium or format, as long as you give appropriate credit to the original author(s) and the source, provide a link to the Creative Commons licence, and indicate if changes were made. The images or other third party material in this article are included in the article's Creative Commons licence, unless indicated otherwise in a credit line to the material. If material is not included in the article's Creative Commons licence and your intended use is not permitted by statutory regulation or exceeds the permitted use, you will need to obtain permission directly from the copyright holder. To view a copy of this licence, visit http://creativecommons.org/licenses/by/4.0/.

\section{References}

1. Boucksom, S., de Fernex, T., Favre, C.: The volume of an isolated singularity. Duke Math. J. 161(8), 1455-1520 (2012)

2. Evertse, J.-H., Ferretti, R.: Diophantine inequalities on smooth projective varieties. Int. Math. Res. Not. 25, 1295-1330 (2002)

3. Evertse, J.-H., Ferretti, R.: A generalization of the subspace theorem with polynomials of higher degree. In: Diophantine Approximation, Development in Mathematics, vol. 16, pp. 175-198. Springer, NewYork (2008)

4. Faltings, G.: A new application of Diophantine approximations. In: Wüstholz, Gisbert (ed.) A Panorama of Number Theory or the View from Baker's Garden (Zürich, 1999), pp. 231-246. Cambridge Univ. Press, Cambridge (2002)

5. Faltings, G., Wüstholz, G.: Diophantine approximations on projective spaces. Invent. Math. 116, 109138 (1994)

6. Hartshorne, R.: Algebraic Geometry. Graduate Texts in Mathematics, No. 52. Springer, New York (1977)

7. He, Y., Ru, M.: A generalized Schmidt subspace theorem for closed subschemes in subgeneral position. J. Number Theory (to appear)

8. Heier, G., Levin, A.: A generalized Schmidt subspace theorem for closed subschemes. Amer. J. Math. 143, 213-226 (2021)

9. Lang, S.: Fundamentals of Diophantine Geometry. Springer, New York (1983)

10. Lazarsfeld, R.: Positivity in Algebraic Geometry I. Springer, Berlin (2004)

11. Levin, A.: Generalizations of Siegel's and Picard's theorems. Ann. Math. (2) 170(2), 609-655 (2009)

12. Quang, Si Duc: A generalization of the subspace theorem for higher degree polynomials in subgeneral position. Int. J. Number Theory 15(04), 775-788 (2019)

13. $\mathrm{Ru}, \mathrm{M} .:$ On a general form of the second main theorem. Trans. Am. Math. Soc. 349, 5093-5105 (1997)

14. Ru, M.: Holomorphic curves into algebraic varieties. Ann. Math. (2) 169(1), 255-267 (2009)

15. Ru, M.: On a general Diophantine inequality. Funct. Approx. Comment. Math. 56, 143-163 (2017)

16. Ru, M., Vojta, P.: A birational Nevanlinna constant and its consequences Amer. J. Math. 142, 957-991 (2020)

17. Serre, J.-P.: Lectures on the Mordell-Weil theorem. Translated from the French and edited by Martin Brown from notes by Michel Waldschmidt, 2nd edn. Friedr. Vieweg \& Sohn, Braunschweig (1990). Aspects of Mathematics, E15

18. Vojta, P.: Diophantine Approximations and Value Distribution Theory. Lecture Notes in Mathematics, vol. 1239. Springer, Berlin (1987)

19. Vojta, P.: On Cartan's theorem and Cartan's conjecture. Amer. J. Math. 119, 1-17 (1997)

20. Vojta, P.: Integral points on subvarieties of semiabelian varieties. I. Invent. Math. 126, 133-181 (1996)

21. Zannier, U.: On the integral points on the complement of ramification-divisors. J. Inst. Math. Jussieu 4(2), 317-330 (2005)

Publisher's Note Springer Nature remains neutral with regard to jurisdictional claims in published maps and institutional affiliations. 